\title{
Regularisation of Mixed Boundary Problems
}

\author{
B.-W. Schulze A. Shlapunov * N. Tarkhanov
}

January 24, 2000

* Supported by the Max-Planck Gesellschaft and the RFFI grant 99-01-00790. 


\begin{abstract}
We show an application of the spectral theorem in constructing approximate solutions of mixed boundary value problems for elliptic equations.
\end{abstract}




\section{Contents}

Introduction 4

1 Selfadjoint operators $\quad 8$

2 Bounded operators $\quad 11$

3 Green function $\quad 12$

$\begin{array}{ll}4 \text { A crack problem } & 15\end{array}$

5 Adjoint operator $\quad 19$

6 The case of constant coefficients $\quad 24$

$\begin{array}{llr}7 & \text { Iterations } & 26\end{array}$

$\begin{array}{llr}8 & \text { Cauchy problem } & 29\end{array}$

9 Zaremba problem $\quad 30$

10 Examples $\quad 33$

$\begin{array}{ll}\text { References } & 35\end{array}$ 


\section{Introduction}

When studying boundary value problems for solutions of an elliptic differential equation $P u=f, P$ being of type $E \rightarrow F$ for some vector bundles $E$ and $F$, one uses any left parametrix of $P$ to reduce the problem to the boundary. A powerful tool for such a reduction is the Green formula for $P$ which brings together the values of $P u$ in a domain $\mathcal{D}$ and the Cauchy data $t(u)$ of $u$ on the boundary of $\mathcal{D}$ to present $u$ in $\mathcal{D}$ modulo smoothing operators.

In case $P$ has a left fundamental solution $\Phi$, the Green formula reads $u=\mathcal{P}_{\mathrm{dl}} t(u)+\mathcal{P}_{\mathrm{v}} P u$ in $\mathcal{D}$, where $\mathcal{P}_{\mathrm{dl}} u_{0}$ and $\mathcal{P}_{\mathrm{v}} f$ are the double layer and volume potentials corresponding to $\Phi$. If moreover $\Phi$ is a right fundamental solution, then the potential $\mathcal{P}_{\mathrm{dl}} u_{0}$ satisfies the homogeneous equation $P u=0$ in $\mathcal{D}$, and so the Green formula provides us with a soft version of the Hodge decomposition.

However, right fundamental solutions are available only for those $P$ which are determined, i.e., bear as many scalar equations as the number of unknown functions. For overdetermined elliptic operators $P$, the construction of kernels $\Phi$ with the property that $P \mathcal{P}_{\mathrm{dl}} u_{0}=0$ in $\mathcal{D}$, for each density $u_{0}$, is a significant problem. In complex analysis such Green formulas are known as Cauchy-Fantappiè formulas. They give rise to explicit solutions of the inhomogeneous Cauchy-Riemann system $\bar{\partial} u=f$ on strongly pseudoconvex manifolds or domains in $\mathbb{C}^{N}$.

Consider the iterations $\mathcal{P}_{\mathrm{dl}}^{N} t(u)$, for $N=1,2, \ldots$, in any function space $H$ invariant under $\mathcal{P}_{\mathrm{dl}}$. If $\Phi$ is a right fundamental solution of $P$, then these iterations stabilise because $P \mathcal{P}_{\mathrm{dl}} t(u)=0$ in $\mathcal{D}$ and so $\mathcal{P}_{\mathrm{dl}}^{N} t(u)=\mathcal{P}_{\mathrm{dl}} t(u)$ for all $N$. In general, since $\mathcal{P}_{\mathrm{dl}}$ is the identity operator on solutions of $P u=0$, one may conjecture that the iterations converge to a projection of $H$ onto the subspace $V_{1}$ of $H$ consisting of $u$ satisfying $P u=0$ in $\mathcal{D}$. Were such the case, the equality

$$
u=\pi_{V_{1}} u+\sum_{\nu=0}^{\infty} \mathcal{P}_{\mathrm{dl}}^{\nu} \mathcal{P}_{\mathrm{v}} P u,
$$

for $u \in H$, would give us a substitute for the Hodge decomposition.

This idea goes back to the work of Romanov [Rom78] who studied the iterations of the Martinelli-Bochner integral in $\mathbb{C}^{N}, N>1$. His results were extended in a beautiful way to arbitrary overdetermined elliptic systems by Nacinovich and Shlapunov [NS96].

To handle the iterations of $\mathcal{P}_{\mathrm{dl}}$, the idea is to present this integral as a selfadjoint operator on a Hilbert space $H$. While $H$ is specified within the Sobolev space $H^{p}$ in $\mathcal{D}, p$ being the order of $P$, the Hermitian structure of $H$ is different from that induced by $H^{p}$. If moreover $0 \leq \mathcal{P}_{\mathrm{dl}} \leq I$, then the iterations of $\mathcal{P}_{\mathrm{dl}}$ converge, by the spectral theorem, to the projection of $H$ onto the eigenspace of $\mathcal{P}_{\mathrm{dl}}$ corresponding to the eigenvalue 1 . This eigenspace in 
turn coincides with the null-space of $\mathcal{P}_{\mathrm{v}} P$ in $H$, and hence with the null-space of $P$ if $\mathcal{P}_{\mathrm{v}}$ is identified with the adjoint of $P$ in $H$.

The choice of $H$ is actually suggested by the restriction of $\Phi$ to the closed domain $\overline{\mathcal{D}}$. If $\mathcal{P}_{\mathrm{v}} f$ meets automatically some conditions on the boundary of $\mathcal{D}$, for each $f \in L^{2}(\mathcal{D}, F)$, these should be incorporated in the definition of $H$.

To introduce the relevant Hermitian structure in $H$, we choose any domain $\mathcal{D}^{\prime}$ with $C^{\infty}$ boundary, such that $\overline{\mathcal{D}} \subset \mathcal{D}^{\prime}$, and we fix an extension operator $e: H \rightarrow H^{p}\left(\mathcal{D}^{\prime}, E\right)$ with the properties that $t(e(u))=0$ on $\partial \mathcal{D}^{\prime}$, for each $u \in H$, and $e\left(\mathcal{P}_{\mathrm{v}} f\right)=\mathcal{P}_{\mathrm{v}} f$, for each $f \in L^{2}(\mathcal{D}, F)$. In this way we actually reduce the problem to that on a compact surrounding manifold. As but one example of $e(u)$ we show the solution of the Dirichlet problem for the Laplacian $\Delta=P^{*} P$ in $\mathcal{D}^{\prime} \backslash \overline{\mathcal{D}}$ with data $t(u)$ on $\partial \mathcal{D}$ and 0 on $\partial \mathcal{D}^{\prime}$. Define a scalar product in $H$ by

$$
h(u, v)=(P e(u), P e(v))_{L^{2}\left(\mathcal{D}^{\prime}, F\right)},
$$

then the identity $h\left(\mathcal{P}_{\mathrm{v}} f, v\right)=(f, P v)_{L^{2}(\mathcal{D}, F)}$, for every $v \in H$, is immediate. It in turn implies the selfadjointness of $\mathcal{P}_{\mathrm{dl}}$ with respect to $(0.2)$.

In [NS96], $H$ is the whole space $H^{p}(\mathcal{D}, E)$, and $\mathcal{P}_{\mathrm{v}} f=\mathcal{G} P^{*}\left(\chi_{\mathcal{D}} f\right)$ where $\mathcal{G}$ is the Green function of the Dirichlet problem for $\Delta$ in $\mathcal{D}^{\prime}$ and $\chi_{\mathcal{D}}$ is the characteristic function of $\mathcal{D}$. In this setting, the orthogonal decomposition (0.1) applies to constructing approximate solutions for the inhomogeneous equation $P u=f$ and the Dirichlet system for the Laplacian $\Delta$ in $\mathcal{D}$.

This work was intended as an attempt to develop the approach of [NS96] to derive approximate solutions of the generalised Zaremba problem in $\mathcal{D}$. It consists of finding a function $u \in H^{p}(\mathcal{D}, E)$ satisfying $\Delta u=f$ in $\mathcal{D}$, whose 'tangential part' $t(u)$ takes prescribed values on a part $\sigma$ of $\partial \mathcal{D}$ while the 'normal part' $n(P u)$ takes prescribed values on the complementary part $\partial \mathcal{D} \backslash \bar{\sigma}$. Since the Dirichlet problem in $\mathcal{D}$ is elliptic, we may assume without loss of generality that $t(u)=0$ on $\sigma$. This causes $H$ to be a subspace of $H^{p}(\mathcal{D}, E)$ whose elements satisfy $t(u)=0$ on $\sigma$. Moreover, $\mathcal{G}$ should be the Green function of the Dirichlet problem in the domain with crack $\mathcal{D}^{\prime} \backslash \bar{\sigma}$.

Thus, the study of the Zaremba problem leads to the Dirichlet problem for the generalised Laplace operator $\Delta$ in a domain with cracks. Note that both mixed boundary value problems and crack problems can be treated in the framework of any calculus on manifolds with edges of codimension 1 on the boundary. At present, several calculi on such singular configurations are known, namely those by Vishik and Eskin (cf. Section 24 in [Esk73]), Maz'ya and Plamenevskii (cf. Chapter 8 in [NP91]), Schulze [Sch92], Duduchava and Wendland [DW95], Rabinovich, Schulze and Tarkhanov [RST98], etc. Either of these calculi is applicable.

The important point to note here is the function spaces used as domains for pseudodifferential operators involved. These are scales of weighted Sobolev spaces $H^{s, \gamma}(\mathcal{D}, E)$ parametrised by $s, \gamma \in \mathbb{R}$, where $s$ specifies the smoothness 
and $\gamma$ stands for the weight. While the spaces may differ from each other in various calculi, they usually coincide with $L^{2}(\mathcal{D}, E)$, for $s, \gamma=0$. As weight functions, one uses the powers of the regularised distance to the set of singularities, here to the edge $\partial \sigma$. Moreover, we may always specify the weight exponent $\gamma$ in such a way that admissible operators of order $m$ map $H^{s, \gamma}(\mathcal{D}, E)$ to $H^{s-m, \gamma-m}(\mathcal{D}, F)$.

Let us dwell upon the contents of the paper. Section 1 presents generalities on bounded selfadjoint operators in Hilbert spaces.

In Section 2 we indicate how these results apply to non-necessarily selfadjoint operators. The idea is to replace the equation $B u=f$ by $B^{*} B u=B^{*} f$ and shrink the domain of $f$ to those which are orthogonal to the null-space of $B^{*}$.

Section 3 discusses in detail the construction of the Green function for the classical Dirichlet problem in a plane domain with a crack along a segment. We make use of layer potentials to present the Green function, thus showing its asymptotics near the boundary points of the segment. This section is intended to motivate our investigation of crack problems.

In Section 4 we formulate the Dirichlet problem for the generalised Laplacian in a domain with a crack along a hypersurface with $C^{\infty}$ boundary. When compared to general crack problems, the Dirichlet problem has the peculiarity of being formally selfadjoint. Moreover, any solution of the corresponding homogeneous problem is trivial unless it is rather singular. Hence it follows that the Dirichlet problem is uniquely solvable provided it is Fredholm. Since the Dirichlet problem is elliptic in the usual sense on the smooth part of the boundary, i.e., away from $\partial \sigma$, the Fredholm property of this problem is equivalent to the bijectivity of an operator-valued symbol called the edge symbol. This symbol lives in the cotangent bundle to $\partial \sigma$ with the zero section removed. In fact, the edge symbol is the Dirichlet problem for a differential operator of special form in the plane with a cut along the non-negative semiaxis. The operator is obtained from $P^{*} P$ by freezing the coefficients at any point $y \in \partial \sigma$ and substituting the covariable $\eta$ for the derivatives along the edge. It is supplied by the Dirichlet boundary conditions from both sides of the cut. Using polar coordinates in the normal plane to the edge at $y$, we see that the conormal symbol of the edge symbol is $\left(\sigma^{p}(P)\right)^{*} \sigma^{p}(P)$, the principal symbol of $P$ being evaluated at $\eta=0$ and

$$
\begin{aligned}
& \xi_{1}=\cos \varphi D_{r}-(1 / r) \sin \varphi D_{\varphi}, \\
& \xi_{2}=\sin \varphi D_{r}+(1 / r) \cos \varphi D_{\varphi},
\end{aligned}
$$

$\xi_{1}, \xi_{2}$ standing for the conormal variables. Thus, if supplied by the Dirichlet conditions at the endpoints of $[0,2 \pi]$, the conormal symbol belongs to the same class of boundary value problems, now on a segment. The condition of bijectivity of the conormal symbol gives us a discrete set $\Sigma$ of "prohibited" 
weights $\gamma$, independent of $\eta$. For $\gamma \notin \Sigma$, the edge symbol is a Fredholm operator in Sobolev spaces of weight $\gamma$ over $\mathbb{R}^{2} \backslash \overline{\mathbb{R}}_{+}$. It is to be expected that the null-space of the edge symbol is trivial if $\gamma \gg 0$ is large enough. By duality, if $-\gamma \gg 0$ is large enough, then the cokernel of the edge symbol is trivial. It is now the property of $P, t$ and the singular configuration whether or not, given a $\gamma$, the edge symbol is an isomorphism for all $\eta \neq 0$. For the Dirichlet problem in a domain with a smooth edge, the edge symbol is known to be an isomorphism for $\gamma$ in an interval around $p$ (see Sections 6.1.3 and 8.4.2 in [NP91]). It follows that the Dirichlet problem for the Laplacian $\Delta=P^{*} P$ is solvable in $H^{s, p}(\mathcal{D}, E), \mathcal{D}$ being a domain with smooth edges. Then, we make use of a familiar classical scheme to construct the Green function of the problem.

Section 5 contains a construction of a scalar product $h(u, v)$ in the space $H^{p, p}(\mathcal{D}, E)$, such that the norm $\sqrt{h(u, u)}$ is equivalent to the original one. If restricted to the subspace $H$ of $H^{p, p}(\mathcal{D}, E)$ which consists of the functions with vanishing Dirichlet data on $\sigma$, this scalar product makes selfadjoint the double layer potential related to the Green function of a larger domain $\mathcal{D}^{\prime}$ with a crack along $\sigma$.

In Section 6 we simplify the construction of $h(u, v)$ in case $P$ is a differential operator with constant coefficients in $\mathbb{R}^{n}$. If $P^{*} P$ has a fundamental solution of convolution type $\mathcal{G}(x)$ which decreases at infinity fast enough, we may think of $\mathcal{G}(x)$ as a Green function of the one-point compactification of $\mathbb{R}^{n}$. Moreover, we can solve the Dirichlet problem for $\mathcal{G}(x-y), y \notin \bar{\sigma}$, with data on $\sigma$, thus arriving at a Green function of $\mathbb{R}^{n} \backslash \bar{\sigma}$. Hence we have a canonical choice for $\mathcal{D}^{\prime}$, namely $\mathcal{D}^{\prime}=\mathbb{R}^{n}$. This works, in particular, for first order homogeneous operators $P$ with constant coefficients.

Section 7 provides a detailed exposition of iteration of the double layer and volume potentials. Let $A$ be a non-negative selfadjoint bounded operator in a Hilbert space $H$, satisfying $\|A\| \leq 1$. By the spectral theorem, we have $A^{N}=\int_{0-}^{1+0} \lambda^{N} d I(\lambda)$ for any $N=1,2, \ldots$, where $I(\lambda)$ is a resolution of the identity in $H$ corresponding to $A$. Hence it follows that the iterations of $A$ converge to the orthogonal projection of $H$ onto the null-space of the complementary operator $I-A$. As but one application of this we show the orthogonal decomposition

$$
I=\lim _{N \rightarrow \infty} A^{N}+\sum_{\nu=0}^{\infty} A^{\nu}(I-A)
$$

in $H$ (cf. [Sh199]). Obviously, (0.1) is a very particular case of this latter formula.

In Section 8 we indicate how (0.1) may be used to yield approximate solutions to the Cauchy problem for $P u=f$ in $\mathcal{D}$ with data on $\sigma$. Write $\pi_{V_{0}}$ for the orthogonal projection of $H$ onto the null-space of $A$. Consider the operator 
$\mathcal{R}=\sum_{\nu=0}^{\infty}(I-A)^{\nu}$ in $H$ with a domain Dom $\mathcal{R}$ consisting of those $f \in H$ for which this series converges. If $f \in \operatorname{Dom} \mathcal{R}$, then $A \mathcal{R} f=f-\pi_{V_{0}} f$. If moreover $f$ is orthogonal to the null-space of $A$, then $A \mathcal{R} f=f$. Conversely, if $f=A u$, for some $u \in H$, then $f \in \operatorname{Dom} \mathcal{R}$ and $f \perp$ ker $A$. On the other hand, the equation $B u=f$, for a non-necessarily selfadjoint operator $B \in \mathcal{L}(H, \tilde{H})$, reduces to $B^{*} B u=B^{*} f$ under the additional condition $f \perp \operatorname{ker} B^{*}$. Assuming $\sigma \neq \emptyset$, we apply this approach to the operator $B=P$ whose adjoint is $\mathcal{P}_{\mathrm{v}}$.

In Section 9 we derive in a similar way an interesting formula for solutions of the generalised Zaremba problem in $\mathcal{D}$, the Dirichlet data being given on $\sigma \neq \emptyset$.

Finally, Section 10 deals with some examples which concern the case where $\sigma$ is an entire component of the boundary surface. The advantage of this situation is that we need not invoke any weighted Sobolev spaces. Using explicit formulas for the Green function of $\mathcal{D}^{\prime} \backslash \bar{\sigma}$ we are able to construct approximate solutions for the Cauchy and Zaremba problems in $\mathcal{D}$ with data on $\sigma$, thus recovering the results of [NS96].

\section{Selfadjoint operators}

Let $H$ be a Hilbert space with a scalar product $(., .)_{H}$ and $A$ be a bounded selfadjoint operator in $H$.

We also assume that $A$ is non-negative, i.e., $(A u, u)_{H} \geq 0$ for every $u \in H$. It is well known that if $H$ is a complex Hilbert space then any non-negative operator is selfadjoint.

We can certainly assume that $\|A\| \leq 1$, since otherwise we replace $A$ by its multiple $A /\|A\|$.

Problem 1.1 Let $f \in H$ be given. Find (if possible) an element $u \in H$ such that $A u=f$.

Generally speaking, Problem 1.1 is ill-posed, i.e., it may happen that the image of $A$ is not closed. This means that no solution exists for some data $f \in H$ and the solution, if exists, do not depend continuously on the data. Hence it follows that the solvability conditions for the problem can not be described in terms of continuous linear functionals.

Let $\operatorname{Id}_{H}$ stand for the identity operator in $H$. If it causes no confusion we write simply Id instead of $\operatorname{Id}_{H}$.

As usual we denote $\operatorname{ker} A$ the null-space of $A$. If $V$ is a closed subspace of $H$, we write $\pi_{V}$ for the orthogonal projection of $H$ onto $V$. 
Theorem 1.2 In the strong operator topology of the space $\mathcal{L}(H)$, the equalities hold

$$
\begin{aligned}
\lim _{N \rightarrow \infty} A^{N} & =\pi_{\operatorname{ker}(I-A)}, \\
\lim _{N \rightarrow \infty}(I-A)^{N} & =\pi_{\operatorname{ker} A} .
\end{aligned}
$$

Proof. Since the operator $A$ is continuous, its kernel $\operatorname{ker}(I-A)$ is a closed subspace of $H$. Therefore, when endowed with the Hermitian structure induced from $H$, it is a Hilbert space.

Given any $N=1,2, \ldots$, the spectral theorem for bounded selfadjoint operators yields

$$
A^{N}=\int_{-0}^{1+0} \lambda^{N} d I(\lambda)
$$

where $(I(\lambda))_{0 \leq \lambda \leq 1}$ is a resolution of the identity in $H$, corresponding to the selfadjoint operator $0 \leq A \leq I$ (see, for instance, Sections 5, 6 in [Yos65, Ch. XI]).

Passing to the limit in (1.1) one obtains

$$
\lim _{N \rightarrow \infty} A^{N}=I(1+0)-I(1-0) .
$$

Since $I(\lambda)$ is a spectral function, the operator $I(1+0)-I(1-0)$ is an orthogonal projection of $H$ onto a closed subspace $V(1)$ in $H$.

Obviously,

$$
(I-A) \lim _{N \rightarrow 0} A^{N} u=0
$$

for every $u \in H$, i.e., $V(1) \subset \operatorname{ker}(I-A)$. Finally, if $u \in \operatorname{ker}(I-A)$ then

$$
\begin{aligned}
u & =A u+(I-A) u \\
& =A u \\
& =A^{N} u,
\end{aligned}
$$

for every $N \geq 0$. Therefore,

$$
u=\lim _{N \rightarrow \infty} A^{N} u
$$

and so $V(1)=\operatorname{ker}(I-A)$ whence

$$
\lim _{N \rightarrow \infty} A^{N}=\pi_{\operatorname{ker}(I-A)} .
$$

In order to complete the proof it suffices to observe that since $A$ is a selfadjoint non-negative operator with $\|A\| \leq 1$, the operator $I-A$ has the same properties. 
Corollary 1.3 In the strong operator topology of the space $\mathcal{L}(H)$, the equalities hold

$$
\begin{aligned}
& I=\lim _{N \rightarrow \infty} A^{N}+\sum_{\nu=0}^{\infty} A^{\nu}(I-A), \\
& I=\lim _{N \rightarrow \infty}(I-A)^{N}+\sum_{\nu=0}^{\infty}(I-A)^{\nu} A .
\end{aligned}
$$

Proof. The equality $A+(I-A)=I$ implies

$$
\begin{aligned}
I & =A^{N}+\sum_{\nu=0}^{N-1} A^{\nu}(I-A) \\
& =(I-A)^{N}+\sum_{\nu=0}^{N-1}(I-A)^{\nu} A,
\end{aligned}
$$

for every $\nu \in \mathbb{N}$. Using Theorem 1.2 we can pass to the limit, when $N \rightarrow \infty$, thus obtaining (1.2).

We use Corollary 1.3 in order to obtain a solvability condition for Problem 1.1.

Theorem 1.4 Problem 1.1 is solvable if and only if the Neumann series

$$
u=\sum_{\nu=0}^{\infty}(I-A)^{\nu} f
$$

converges in H. Moreover, if Problem 1.1 is solvable then $A u=f$.

Proof. Let Problem 1.1 have a solution $\tilde{u} \in H$. Then Corollary 1.3 implies that the series

$$
\begin{aligned}
\sum_{\nu=0}^{\infty}(I-A)^{\nu} A \tilde{u} & =\sum_{\nu=0}^{\infty}(I-A)^{\nu} f \\
& =u
\end{aligned}
$$

converges in $H$.

Conversely, let the series $u$ converge in $H$. Since the operator $I-A$ is continuous it follows that

$$
\begin{aligned}
u-A u & =(I-A) u \\
& =\sum_{\nu=1}^{\infty}(I-A)^{\nu} f \\
& =u-f .
\end{aligned}
$$

Hence $A u=f$ and Problem 1.1 is solvable.

Theorem 1.2 implies that the solution $u$ of Theorem 1.4 is a unique solution to Problem 1.1 orthogonal to $\operatorname{ker} A$. 
Remark 1.5 Clearly, the elements

$$
u^{(N)}=\sum_{\nu=0}^{N-1}(I-A)^{\nu} f
$$

can be regarded as approximate solutions to Problem 1.1, even if no real solution exists.

\section{Bounded operators}

Consider now a more general situation. Let $H, \tilde{H}$ be Hilbert spaces, and $B \in \mathcal{L}(H, \tilde{H})$ a bounded operator. Without loss of generality we again restrict our attention to those $B$ which satisfy $\|B\| \leq 1$.

Problem 2.1 Let $f \in \tilde{H}$ be given. Find an element $u \in H$ such that $B u=f$.

Let $B^{*}: \tilde{H} \rightarrow H$ be the adjoint of $B: H \rightarrow \tilde{H}$ in the sense of Hilbert spaces.

Lemma 2.2 Problem 2.1 is solvable if and only if

1) there is a $u \in H$ such that $B^{*} B u=B^{*} f$;

2) $(f, g)_{\tilde{H}}=0$ for all $g \in \operatorname{ker} B^{*}$.

Proof. Indeed, from $B u=f$ it follows readily that $B^{*} B u=B^{*} f$. Conversely, if

$$
\begin{aligned}
B^{*} B u & =B^{*} f, \\
f & \perp \operatorname{ker} B^{*},
\end{aligned}
$$

then $B^{*}(B u-f)=0$ whence

$$
\begin{aligned}
(B u-f, B u-f)_{\tilde{H}} & =(B u, B u-f)_{\tilde{H}}-(f, B u-f)_{\tilde{H}} \\
& =0
\end{aligned}
$$

i.e., $B u=f$.

It is easy to see that $A=B^{*} B$ is a bounded non-negative self-adjoint operator in $H$ satisfying $\|A\| \leq 1$. Therefore Problem 2.1 is equivalent to Problem 1.1, with $f$ replaced by $B^{*} f, f$ being orthogonal to the null-space of $B^{*}$. 
Theorem 2.3 In the strong operator topology of the space $\mathcal{L}(\tilde{H})$, the equality holds

$$
\pi_{\left(\operatorname{ker} B^{*}\right)^{\perp}}=\sum_{\nu=0}^{\infty} B\left(I-B^{*} B\right)^{\nu} B^{*} .
$$

Proof. It is easy to see that

$$
\left(\operatorname{Id}_{\tilde{H}}-B B^{*}\right) B=B\left(\operatorname{Id}_{H}-B^{*} B\right)
$$

whence

$$
\left(\operatorname{Id}_{\tilde{H}}-B B^{*}\right)^{\nu} B B^{*}=B\left(\operatorname{Id}_{H}-B^{*} B\right)^{\nu} B^{*}
$$

for all $\nu>0$.

Note that $B B^{*}$ is a bounded selfadjoint non-negative operator in $\tilde{H}$ satisfying $\left\|B B^{*}\right\| \leq 1$. According to Theorem 1.2 and Corollary 1.3, we get

$$
\begin{aligned}
\pi_{\left(\operatorname{ker} B^{*}\right)^{\perp}} & =\sum_{\nu=0}^{\infty}\left(\operatorname{Id}_{\tilde{H}}-B B^{*}\right)^{\nu} B B^{*} \\
& =\sum_{\nu=0}^{\infty} B\left(\operatorname{Id}_{H}-B^{*} B\right)^{\nu} B^{*}
\end{aligned}
$$

as desired.

Corollary 2.4 Problem 2.1 is solvable if and only if

1) the series $u=\sum_{\nu=0}^{\infty}\left(I-B^{*} B\right)^{\nu} B^{*} f$ converges in $H$;

2) $(f, g)_{\tilde{H}}=0$ for all $g \in \operatorname{ker} B^{*}$.

Moreover, if Problem 2.1 is solvable then the series $u$ is one of its solutions.

Proof. This follows immediately from Lemma 2.2 and Theorems 1.4 and 2.3 .

In the sequel we will discuss some applications of this approach to elliptic equations (cf. also [Sh199]).

\section{Green function}

Fix an interval $\sigma=(a, b)$ in the real axis. Consider the following Dirichlet problem in the plane with a crack along $\sigma$. Given any $u_{0} \in C_{\text {loc }}(\sigma)$, find a harmonic function $u$ in $\mathbb{R}^{2} \backslash \bar{\sigma}$, such that $u$ extends continuously to $\sigma$ from both the upper and lower half-planes and $u^{ \pm}=u_{0}$ on $\sigma$. 
We look for a solution of this problem by the layer potentials method. To this end, denote by

$$
G(x)=\frac{1}{2 \pi} \log |x|
$$

the standard fundamental solution of convolution type to the Laplace operator in the plane. Assuming $u$ to have at $a$ and $b$ poles of at most finite order, we conclude that $u$ extends to a distribution on $\mathbb{R}^{2}$ whence

$$
u(x)=u_{e}(x)+\int_{\sigma}\left(\left(\partial / \partial y_{2}\right) G(x-y) U_{0}(y)-G(x-y) U_{1}(y)\right) d y_{1},
$$

for $x \notin \bar{\sigma}$, where $u_{e}$ is a harmonic function on all of $\mathbb{R}^{2}$, and $U_{0}, U_{1} \in \mathcal{E}^{\prime}(\mathbb{R})$ are supported on $\bar{\sigma}$.

When crossing the interval $\sigma$, the double layer potential has the jump $U_{0}\left(x^{0}\right)$ at each Lebesgue point $x^{0} \in \sigma$ of $U_{0}$. Since we require $u$ to be continuous up to $\sigma$, it follows that $U_{0}$ should vanish on $\sigma$. Hence $U_{0}$ is a finite linear combination of the derivatives of the Dirac delta-functions supported at $a$ and b. Substituting this into (3.1) gives

$$
\begin{aligned}
u(x) & =u_{e}(x) \\
& +\sum_{j=0}^{J(a)} c_{j}(a)\left(\frac{\partial}{\partial x_{1}}\right)^{j} \frac{1}{2 \pi} \frac{-x_{2}}{\left(x_{1}-a\right)^{2}+x_{2}^{2}}+\sum_{j=0}^{J(b)} c_{j}(b)\left(\frac{\partial}{\partial x_{1}}\right)^{j} \frac{1}{2 \pi} \frac{-x_{2}}{\left(x_{1}-b\right)^{2}+x_{2}^{2}} \\
& -\int_{\sigma} G(x-y) U_{1}(y) d y_{1},
\end{aligned}
$$

for $x \notin \bar{\sigma}$.

The second term on the right hand side of this formula is a harmonic function in $\mathbb{R}^{2} \backslash\{a\}$, vanishing both on $\sigma$ and at the point at infinity. The third term bears the same properties, with $a$ replaced by $b$. Hence, to achieve the uniqueness in our problem, we need to impose additional conditions on the behaviour of $u$ near the boundary of $\sigma$. They can be formulated in terms of belonging of $u$ to weighted Sobolev spaces near the singular points, namely $u \in H^{s, \gamma}(B \backslash \bar{\sigma}), \gamma \geq 0$. Here, $B$ is any disk in the plane containing $\bar{\sigma}$, and $H^{s, \gamma}(B \backslash \bar{\sigma})$, for $s \in \mathbb{Z}_{+}$, is the space of all functions $u \in H_{\mathrm{loc}}^{s, \gamma}(B \backslash \bar{\sigma})$ with finite norm

$$
\|u\|_{H^{s, \gamma}(B \backslash \bar{\sigma}, E)}=\left(\int_{B \backslash \bar{\sigma}} \sum_{|\alpha| \leq s} \min (|x-a|,|x-b|)^{2(|\alpha|-\gamma)}\left|D^{\alpha} u(x)\right|^{2} d x\right)^{1 / 2} .
$$

Further, the function $u_{e}$ is not uniquely determined by the data of the problem, too. Indeed, $u_{e}(x)=x_{2}$ is harmonic on all of $\mathbb{R}^{2}$ and vanishes on $\sigma$. To specify $u_{e}$ we impose on $u$ the condition that $u(x)$ has a limit as $|x| \rightarrow \infty$. 
Denoting this limit by $c$, we see at once $u_{e}(x) \equiv c$, for the limit of the integral $\int_{\sigma} G(x-\cdot) U_{1} d y_{1}$, as $|x| \rightarrow \infty$, coincides with that of a constant multiple of $G(x)$. To show this, observe that the difference $G(x-y)-G(x)$ tends to 0 , when $|x| \rightarrow \infty$, the limit being uniform in $y \in \sigma$.

The simple layer potential of a continuous density on $\sigma$ is a continuous function away from the endpoints of $\sigma$ in $\mathbb{R}^{2}$. From what has already been proved it follows that a function $u$ of the form (3.1) is a solution to our crack problem if and only if $u_{e}(x) \equiv c, U_{0}(x) \equiv 0$ and the density $U_{1}$ satisfies the equation

$$
u_{0}(t)=c-\int_{a}^{b} \frac{1}{2 \pi} \log |t-s| U_{1}(s) d s, \quad t \in(a, b) .
$$

Note that (3.2) is a Fredholm equation of the first kind, whose kernel has a weak singularity on the diagonal. It was Carleman [C22] who first solved the equation (3.2) in a closed form. Assume that $u_{0}$ is a $C^{1}$ function on $(a, b)$ and the derivative of $u_{0}$ is of the form $f(t) /(t-a)^{1-\varepsilon}(b-t)^{1-\varepsilon}$, with $\varepsilon>0, f$ satisfying a Hölder condition on $(a, b)$. We look for a solution to (3.2) of the form $U(s) /(s-a)^{1-\varepsilon}(b-s)^{1-\varepsilon}$, where $\varepsilon>0$ and $U$ meets a Hölder condition on $(a, b)$. Then, the result of Carleman [C22] reads as follows.

\section{Lemma 3.1}

1) If $b-a \neq 4$, then, for each $u_{0}$, the equation (3.2) has a unique solution given by

$$
\begin{aligned}
U_{1}(s) & =\frac{2}{\pi} \frac{1}{\sqrt{(s-a)(b-s)}} \\
\times & \left(\int_{a}^{b} \frac{\sqrt{(t-a)(b-t)}}{s-t} u_{0}^{\prime}(t) d t+\frac{1}{\log \frac{b-a}{4}} \int_{a}^{b} \frac{c-u_{0}(t)}{\sqrt{(t-a)(b-t)}} d t\right) .
\end{aligned}
$$

2) If $b-a=4$, then in order that (3.2) be solvable it is necessary and sufficient that $\int_{a}^{b} \frac{c-u_{0}(t)}{\sqrt{(t-a)(b-t)}} d t=0$. Under this latter condition, we have

$$
U_{1}(s)=\frac{2}{\pi} \frac{1}{\sqrt{(s-a)(b-s)}}\left(\int_{a}^{b} \frac{\sqrt{(t-a)(b-t)}}{s-t} u_{0}^{\prime}(t) d t+C\right)
$$

where $C$ is an arbitrary constant.

Proof. See ibid. and Section 55 in Gakhov [G77]. 
By the very construction of reducing (3.2) to a singular integral equation with the Cauchy kernel on $\sigma$, the solution is obtained in the form $U_{1}(s)=U^{\prime}(s)$, with $U(a)=U(b)=0$ (cf. (55.24) in [G77]). Hence it follows that the integral $\int_{\sigma} G(x-y) U_{1}(y) d y_{1}=\left.G\left(x-\left(y_{1}, 0\right)\right) U\left(y_{1}\right)\right|_{y_{1}=a} ^{y_{1}=b}+\int_{\sigma}\left(\partial / \partial x_{1}\right) G(x-y) U(y) d y_{1}$ tends to zero, when $|x| \rightarrow \infty$. Thus, if $b-a \neq 4$, then the unique solution to the Dirichlet problem in $\mathbb{R}^{2} \backslash \bar{\sigma}$ with the data $u_{0}$ on $\sigma$ and $c$ at the point at infinity is given by

$$
u(x)=c-\int_{\sigma} G(x-y) U_{1}(y) d y_{1}, \quad x \in \mathbb{R}^{2} \backslash \bar{\sigma}
$$

the density $U_{1}$ being defined in Lemma 3.1. As explained above, this solution is the best of many other possible solutions.

Using the explicit solution of the Dirichlet problem in $\mathbb{R}^{2} \backslash \bar{\sigma}$, we can construct the Green function of this domain. To this end, fix any point $y \in \mathbb{R}^{2} \backslash \bar{\sigma}$ and denote by $R(\cdot, y)$ the unique harmonic function in $\mathbb{R}^{2} \backslash \bar{\sigma}$, such that $R(x, y)=G(x-y)$ on $\sigma$ and $R(x, y) \rightarrow 0$ as $|x| \rightarrow \infty$. From (3.3) we deduce that $R(x, y)$ is a $C^{\infty}$ function of the parameter $y$ with values in $C_{\text {loc }}^{\infty}\left(\mathbb{R}^{2} \backslash \bar{\sigma}\right)$. The difference $\mathcal{G}(x, y)=G(x-y)-R(x, y)$ is the desired Green function of $\mathbb{R}^{2} \backslash \bar{\sigma}$. For $x \neq y, \mathcal{G}(x, y)$ is $C^{\infty}$ up to $\sigma$ from both sides of this interval, hence the only singularities of $\mathcal{G}(x, y)$ away from the diagonal lie at the corner points $\partial \sigma$. In fact, we have

$$
\mathcal{G}(x, y)=G(x-y)+\int_{a}^{b} G(x-s) \frac{\partial}{\partial s}\left(\frac{2}{\pi} Z(s) \int_{a}^{b} \frac{\delta(t, y)}{Z(t)} \frac{d t}{t-s}\right) d s,
$$

for $x, y \in \mathbb{R}^{2} \backslash \bar{\sigma}$, where any point $s \in(a, b)$ is identified with the point $(s, 0) \in \mathbb{R}^{2}$ and

$$
\begin{aligned}
Z(s) & =\sqrt{(s-a)(b-s)} \\
\delta(t, y) & =G(t-y)-\frac{1}{\pi} \frac{\log (b-t)}{\log \frac{b-a}{4}} \int_{a}^{b} \frac{G(s-y)}{Z(s)} d s .
\end{aligned}
$$

By (3.4), it is possible to get an explicit estimate of $\mathcal{G}(x, y)$ near the corner points $a$ and $b$. However, for our purposes it will be sufficient to know that $\mathcal{G}(x, y)$ belongs to the space $H^{\infty, 0}(B \backslash \bar{\sigma})$ with respect to either of the variables $x$ and $y$ while the other variable lies away from $B, B$ being a disk containing $\bar{\sigma}$.

Remark 3.2 The Green function $\mathcal{G}(x, y)$ allows one to construct the solution to the Dirichlet problem in $\mathbb{R}^{2} \backslash \bar{\sigma}$ by

$$
u(x)=c+\left(\int_{\sigma} \frac{\partial}{\partial y_{2}} \mathcal{G}(x, \cdot)^{+}\left(u^{+}-c\right) d s-\int_{\sigma} \frac{\partial}{\partial y_{2}} \mathcal{G}(x, \cdot)^{-}\left(u^{-}-c\right) d s\right),
$$


where $u^{ \pm}$are the prescribed limit values of $u$ on $\sigma$ from the upper and lower half-planes, respectively, and $c$ is the prescribed limit of $u(x)$ when $|x| \rightarrow \infty$.

\section{A crack problem}

Let $X$ be an open set in $\mathbb{R}^{n}$ and $P \in \operatorname{Diff}^{p}(X ; E, F)$ an elliptic differential operator of order $p$ on $X$, where $E=X \times \mathbb{C}^{k}, F=X \times \mathbb{C}^{l}$.

Having fixed Hermitian metrics on $E$ and $F$, denote by $P^{*} \in \operatorname{Diff}^{p}(X ; F, E)$ the formal adjoint for $P$. Then, $\Delta=P^{*} P$ is an elliptic differential operator of order $2 p$ and type $E \rightarrow E$.

Assume that $\Delta$ bears the uniqueness property for the Cauchy problem in the small on $X$. Then it has a two-sided fundamental solution $G$ on $X$.

Let $\mathcal{D}$ be a relatively compact domain in $X$. We emphasise that the boundary of $\mathcal{D}$ need not be smooth. Denote by $\mathcal{S}_{P}(\mathcal{D})$ the space of all solutions of the system $P u=0$ in $\mathcal{D}$.

In Section 5 we construct a special scalar product $h(\cdot, \cdot)$ on the weighted Sobolev space $H^{p, p}(\mathcal{D}, E)$. This is obtained by using a fundamental solution of $\Delta$ having special properties at the boundary of a larger domain $\mathcal{O} \Subset X$ with singular boundary.

Throughout this section we will assume that the boundary of $\mathcal{D}$ consists of two smooth pieces, namely $\partial \mathcal{D}=\sigma \cup(\partial \mathcal{D} \backslash \sigma)$ where $\sigma$ is an open connected subset of $\partial \mathcal{D}$ with smooth boundary. Fix a domain $\mathcal{D}^{\prime}$ with $C^{\infty}$ boundary, such that $\mathcal{D} \Subset \mathcal{D}^{\prime} \Subset X$.

Let we be given a Dirichlet system $\left\{B_{j}\right\}_{j=0}^{p-1}$ of order $(p-1)$ on the smooth part of $\partial \mathcal{D} \cup \partial \mathcal{D}^{\prime}$, with $B_{j}$ being of type $E \rightarrow F_{j}$ and order $m_{j}$. Denote by $\left\{C_{j}\right\}_{j=0}^{p-1}$ the Dirichlet system adjoint to $\left\{B_{j}\right\}$ with respect to the Green formula for $P$ (cf. [Tar95, 9.2.1]). As is well-known, $C_{j}$ is of type $F^{*} \rightarrow F_{j}^{*}$ and order $p-1-m_{j}$. The only singularities of the coefficients of $\left\{C_{j}\right\}$ lie on the interface $\partial \sigma \subset \partial \mathcal{D}$.

By the above, there is a Green operator $G_{P}$ for $P$ whose restriction to the smooth part of $\partial \mathcal{D} \cup \partial \mathcal{D}^{\prime}$ is $G_{P}\left(*_{F} g, u\right)=(t(u), n(g))_{y} d s$, where $d s$ is the area form of $\partial\left(\mathcal{D}^{\prime} \backslash \overline{\mathcal{D}}\right)$ and

$$
\begin{aligned}
& t(u)=\bigoplus_{\substack{j=0 \\
p-1}}^{p-1} B_{j} u, \\
& n(g)=\bigoplus_{j=0}^{p-1} *_{F_{j}}^{-1} C_{j} *_{F} g,
\end{aligned}
$$

for $u \in C^{\infty}(E)$ and $g \in C^{\infty}(F)$. Moreover, $*_{F}$ and $*_{F_{j}}$ are sesquilinear bundle isomorphisms $F \rightarrow F^{*}$ and $F_{j} \rightarrow F_{j}^{*}$ induced by the Hermitian metrics on $F$ and $F_{j}$, respectively. 
Recall that $H^{s, \gamma}(\mathcal{D}, E)$ stands for the weighted Sobolev space of sections of $E$ over $\mathcal{D}$ of smoothness $s$ and weight $\gamma$, both $s$ and $\gamma$ being real numbers. For $s \in \mathbb{Z}_{+}$, it coincides with the completion of the $C^{\infty}$ sections of $E$ vanishing near $\partial \sigma$, with respect to the norm

$$
\|u\|_{H^{s, \gamma}(\mathcal{D}, E)}=\left(\int_{\mathcal{D}} \sum_{|\alpha| \leq s} \operatorname{dist}(x, \partial \sigma)^{2(|\alpha|-\gamma)}\left|D^{\alpha} u(x)\right|^{2} d x\right)^{1 / 2} .
$$

We denote by $H^{s-\frac{1}{2}, \gamma-\frac{1}{2}}(\partial \mathcal{D}, E)$ the space consisting of the traces of functions $u \in H^{s, \gamma}(\mathcal{D}, E)$ on the smooth part of $\partial \mathcal{D}$. As usual, we endow this space by the quotient norm; an inner description is available, too.

In his edge calculus, Schulze [Sch98] uses another scale of weighted Sobolev spaces, denoted by $W^{s, \gamma}(\mathcal{D}, E)$. While $H^{s, \gamma}(\mathcal{D}, E)$ and $W^{s, \gamma}(\mathcal{D}, E)$ are quite different from each other, for arbitrary $s$ and $\gamma$, they coincide on the diagonal $s=\gamma$, i.e., $H^{s, s}(\mathcal{D}, E)=W^{s, s}(\mathcal{D}, E)$ for all $s \in \mathbb{Z}_{+}$(cf. Proposition 3.1.5 in Schulze [Sch99]). This is just the case in the present paper where we deal, for the most part, with the spaces $H^{p, p}(\mathcal{D}, E)$.

Lemma 4.1 Let $\gamma \in \mathbb{R}$. For each $u \in H^{p, \gamma}(\mathcal{D}, E)$ and $g \in H^{p, p-\gamma}(\mathcal{D}, F)$, we have

$$
\int_{\partial \mathcal{D}}(t(u), n(g))_{x} d s=\int_{\mathcal{D}}\left((P u, g)_{x}-\left(u, P^{*} g\right)_{x}\right) d x
$$

Proof. Indeed, if $g=g_{\nu}$ vanishes near $\partial \sigma$, then the equality is simply a Green formula for $P$ (cf. [Tar95, 9.2.1]). Since

$$
\left|\int_{\partial \mathcal{D}}\left(t(u), n\left(g_{\nu}\right)\right)_{x} d s\right| \leq c\|u\|_{H^{p-1, \gamma-\frac{1}{2}(\partial \mathcal{D}, E)}}\left\|g_{\nu}\right\|_{H^{p-1, p-\gamma-\frac{1}{2}(\partial \mathcal{D}, F)}}
$$

and

$$
\begin{aligned}
& \left|\int_{\mathcal{D}}\left(P u, g_{\nu}\right)_{x} d x\right| \leq c\|u\|_{H^{p, \gamma}(\mathcal{D}, E)}\left\|g_{\nu}\right\|_{H^{0, p-\gamma}(\mathcal{D}, F)}, \\
& \left|\int_{\mathcal{D}}\left(u, P^{*} g_{\nu}\right)_{x} d x\right| \leq c\|u\|_{H^{0, \gamma}(\mathcal{D}, E)}\left\|g_{\nu}\right\|_{H^{p, p-\gamma}(\mathcal{D}, F)},
\end{aligned}
$$

with $c$ a constant independent of $u$ and $g_{\nu}$, the proof is completed by a passage to the limit in the formula for $g_{\nu}$ vanishing near $\partial \sigma$ and approximating an arbitrary $g \in H^{p, p-\gamma}(\mathcal{D}, F)$.

Consider the following Dirichlet problem for the Laplacian $\Delta=P^{*} P$ in the domain $\mathcal{O}=\mathcal{D}^{\prime} \backslash \bar{\sigma}$ having a crack along $\bar{\sigma}$. Let $s \in \mathbb{Z}_{+}$satisfy $s \geq p$, and 
let $\gamma \in \mathbb{R}$. Given any $u_{0} \in \oplus H^{s-m_{j}-\frac{1}{2}, \gamma-m_{j}-\frac{1}{2}}\left(\partial \mathcal{O}, F_{j}\right)$, find a $u \in H^{s, \gamma}(\mathcal{O}, E)$ such that

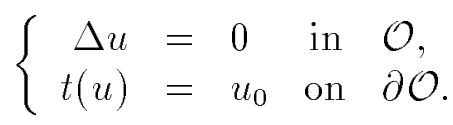

Since $\left\{B_{j}\right\}_{j=0}^{p-1}$ is a Dirichlet system of order $(p-1)$, we may solve (4.1) first in the class of weighted Sobolev spaces, thus reducing the problem to that of finding a $\tilde{u} \in H^{s, \gamma}(\mathcal{O}, E)$ satisfying $\Delta \tilde{u}=f$ in $\mathcal{O}$ and $t(\tilde{u})=0$ on $\partial \mathcal{O}$, for given $f \in H^{s-2 p, \gamma-2 p}(\mathcal{O}, E)$. The advantage of using this reduction lies in the fact that the latter problem can be posed as a variational one. Namely, given any $f \in H^{s-2 p, \gamma-2 p}(\mathcal{O}, E), \gamma \geq p$, find a $\tilde{u} \in H^{s, \gamma}(\mathcal{O}, E)$ such that $t(\tilde{u})=0$ on $\partial \mathcal{O}$ and

$$
\int_{\mathcal{O}}(P \tilde{u}, P v)_{x} d x=\int_{\mathcal{O}}(f, v)_{x} d x
$$

for all $v \in H^{s, \gamma}(\mathcal{O}, E)$ satisfying $t(v)=0$ on $\partial \mathcal{O}$. Note that the restriction $\gamma \geq p$ is necessary because otherwise the pairings in (4.2) are not defined.

If $s=\gamma=p$, then (4.2) is uniquely solvable for all $f \in H^{-p,-p}(\mathcal{O}, E)$. Indeed, denote by $H$ the subspace of $H^{p, p}(\mathcal{O}, E)$ consisting of all $u$ satisfying $t(u)=0$ on $\partial \mathcal{O}$. The sesqui-linear form $(u, v)_{H}=\int_{\mathcal{O}}(P u, P v)_{x} d x$ is easily seen to define a scalar product on $H$. Moreover, the norm $u \mapsto \sqrt{(u, u)_{H}}$ on $H$ is equivalent to that induced by $H^{p, p}(\mathcal{O}, E)$, hence $H$ is Hilbert. For every $f \in H^{-p,-p}(\mathcal{O}, E)$, the functional $v \mapsto \int_{\mathcal{O}}(f, v)_{x} d x$ is continuous on $H$. By the Riesz theorem, this functional can be written in the form (4.2) with some $u \in H$, as desired.

That we have chosen $s=p$ is not important at all, for the Dirichlet problem meets the Lopatinskii condition on the smooth part of $\partial \mathcal{O}$. However, the exponent $\gamma=p$ is of exceptional character. More precisely, the following is true.

Lemma 4.2 There is a closed set $\Sigma \subset \mathbb{R}$ with the property that, if $\gamma \notin \Sigma$, then the Dirichlet problem (4.1) has a unique solution $u \in H^{s, \gamma}(\mathcal{O}, E)$, for each data $u_{0} \in \oplus H^{s-m_{j}-\frac{1}{2}, \gamma-m_{j}-\frac{1}{2}}\left(\partial \mathcal{O}, F_{j}\right)$. Moreover, $p \notin \Sigma$.

Proof. Indeed, (4.1) is an elliptic boundary value problem in the domain $\mathcal{D}^{\prime}$ with a crack along the smooth surface $\sigma$. It can be treated in the framework of analysis on manifolds with boundary and edges of codimension 1 on the boundary (cf. Nazarov and Plamenevskii [NP91], Schulze [Sch92], Duduchava and Wendland [DW95], Rabinovich, Schulze and Tarkhanov [RST98], etc.). Either of these calculi is applicable. The fact that $p \notin \Sigma$ is a consequence of the formal selfadjointness of the Dirichlet problem (see Sections 6.1.3 and 8.4.2 in [NP91]). 
In particular, we are able to invoke the classical scheme of constructing the Green function of an elliptic boundary value problem by improving a two-sided fundamental solution, the domain being $\mathcal{O}=\mathcal{D}^{\prime} \backslash \bar{\sigma}$.

Proposition 4.3 Suppose $1 \notin \Sigma$. Then, there exists a Green function $\mathcal{G}(x, y)$ of problem (4.1), i.e., a two-sided fundamental solution of $\Delta$ in $\mathcal{O}$, such that

1) $\mathcal{G}$ extends to a smooth matrix-valued function away from the diagonal in $\left(\overline{\mathcal{D}}^{\prime} \backslash \partial \sigma\right) \times\left(\overline{\mathcal{D}}^{\prime} \backslash \partial \sigma\right)$, the smoothness near $\sigma \times \sigma$ being understood sidewise;

2) $t(\mathcal{G}(\cdot, y))=0$ on $\partial \mathcal{D}^{\prime} \cup \sigma$, for each $y \in \mathcal{O}$, the operator $t$ acting in the variable $x$.

Proof. It is easy to verify that, given any fixed $y \in \mathcal{O}$, the function $u_{0}=t(G(\cdot, y))$ belongs to $\oplus H^{\infty, \gamma-m_{j}-\frac{1}{2}}\left(\partial \mathcal{D}^{\prime} \cup \sigma, F_{j} \otimes E_{y}^{*}\right)$ for all $\gamma<1$. Denote by $R(\cdot, y)$ the unique solution of problem (4.1) corresponding to this $u_{0}$. Then $\mathcal{G}(x, y)=G(x, y)-R(x, y)$ bears all the desired properties.

If multiplied by an excision function of $y$, the Green function $\mathcal{G}(\cdot, y)$ actually belongs to $H^{\infty, p}\left(\mathcal{O}, E \otimes E_{y}^{*}\right)$, for each $y \in \mathcal{O}$, as is seen from the proof of Proposition 4.3. For more details we refer the reader to Section 6 of [NP91, Ch. 8].

When compared to the explicit formula (3.4), the proof highlights the structure of $\mathcal{G}$. Indeed, $\mathcal{G}$ can be constructed within the pseudodifferential calculus on $\mathcal{O}$ developed in Schulze [Sch98], where $\mathcal{O}$ is thought of as a manifold with edge $\partial \sigma$ on the boundary. Thus, $\mathcal{G}$ inherits the structure of operators in this calculus, cf. Section 3.4 ibid..

Remark 4.4 Since the problem (4.1) is formally selfadjoint, the Green function $\mathcal{G}$ actually satisfies $\mathcal{G}(x, y)^{*}=\mathcal{G}(y, x)$ on $\mathcal{O} \times \mathcal{O}$. However, we will not use this symmetry.

\section{Adjoint operator}

Let $\mathcal{S}_{\Delta}\left(\hat{\mathcal{D}}^{\prime} \backslash \overline{\mathcal{D}}\right)$ be the set of all solutions to the equation $\Delta u=0$ in $\mathcal{D}^{\prime} \backslash \overline{\mathcal{D}}$, such that $u$ has finite order of growth near $\partial \mathcal{D}^{\prime}$ and $t(u)=0$ on $\partial \mathcal{D}^{\prime}$.

Using Lemma 4.2 for $\mathcal{D}^{\prime} \backslash \overline{\mathcal{D}}$ instead of $\mathcal{D}^{\prime} \backslash \bar{\sigma}$, we obtain readily a linear isomorphism

$$
H^{p, \gamma}\left(\mathcal{D}^{\prime} \backslash \overline{\mathcal{D}}, E\right) \cap \mathcal{S}_{\Delta}\left(\hat{\mathcal{D}}^{\prime} \backslash \overline{\mathcal{D}}\right) \stackrel{t_{+}}{\longrightarrow} \oplus H^{p-m_{j}-\frac{1}{2}, \gamma-m_{j}-\frac{1}{2}}\left(\partial \mathcal{D}, F_{j}\right)
$$


given by $\left.u \mapsto t(u)\right|_{\partial \mathcal{D}}$. Finally, composing the inverse $t_{+}^{-1}$ with the trace operator

$$
H^{p, \gamma}(\mathcal{D}, E) \stackrel{t_{-}}{\longrightarrow} \oplus H^{p-m_{j}-\frac{1}{2}, \gamma-m_{j}-\frac{1}{2}}\left(\partial \mathcal{D}, F_{j}\right)
$$

yields a continuous linear mapping

$$
H^{p, \gamma}(\mathcal{D}, E) \stackrel{\mathcal{E}}{\longrightarrow} H^{p, \gamma}\left(\mathcal{D}^{\prime} \backslash \overline{\mathcal{D}}, E\right) \cap \mathcal{S}_{\Delta}\left(\hat{\mathcal{D}}^{\prime} \backslash \overline{\mathcal{D}}\right) .
$$

Suppose $\gamma \geq p$. Then we have $P u \in L^{2}(\mathcal{D}, F)$ and $P \mathcal{E}(u) \in L^{2}\left(\mathcal{D}^{\prime} \backslash \overline{\mathcal{D}}, E\right)$ for each $u \in H^{p, \gamma}(\mathcal{D}, E)$. Consider the Hermitian form

$$
h(u, v)=\int_{\mathcal{D}}(P u, P v)_{x} d x+\int_{\mathcal{D}^{\prime} \backslash \overline{\mathcal{D}}}(P \mathcal{E}(u), P \mathcal{E}(v))_{x} d x
$$

defined for $u, v \in H^{p, \gamma}(\mathcal{D}, E)$.

Proposition 5.1 The Hermitian form $h(\cdot, \cdot)$ defines a scalar product in $H^{p, \gamma}(\mathcal{D}, E)$.

Proof. It suffices to prove that $h(u, u)=0$ implies $u \equiv 0$ in $D$. To do this, pick a $u \in H^{p, \gamma}(\mathcal{D}, E)$ with $h(u, u)=0$. Then, $u \in \mathcal{S}_{P}(\mathcal{D})$ and $\mathcal{E}(u) \in \mathcal{S}_{P}\left(\mathcal{D}^{\prime} \backslash \overline{\mathcal{D}}\right)$. By the construction of $\mathcal{E}$, we have $t(u)=t(\mathcal{E}(u))$ on the smooth part of the boundary of $\mathcal{D}$. According to Theorem 1.3.3 in [Tar97] there is a solution $\tilde{u} \in \mathcal{S}_{P}\left(\mathcal{D}^{\prime} \backslash \partial \sigma\right)$ such that $\tilde{u}=u$ in $\mathcal{D}$ and $\tilde{u}=\mathcal{E}(u)$ in $\mathcal{D}^{\prime} \backslash c \bar{D}$. In particular, $t(\tilde{u})=0$ on $\partial \mathcal{D}^{\prime}$. Since the uniqueness property holds true for the Cauchy problem in the small for the Laplacian $P^{*} P$, it does so for $P$. This gives $\tilde{u} \equiv 0$ on all of $\mathcal{D}^{\prime} \backslash \partial \sigma$ (cf., for instance, [Tar95, 10.3.1]). In particular, $u=0$ in $\mathcal{D}$, as desired.

Set

$$
\Phi(x, y)=*_{F} P(y, D) *_{E}^{-1} \mathcal{G}(x, y),
$$

for $(x, y) \in\left(\mathcal{D}^{\prime} \backslash \bar{\sigma}\right) \times\left(\mathcal{D}^{\prime} \backslash \bar{\sigma}\right)$. Moreover, introduce the double layer and volume potentials by

$$
\begin{aligned}
-\mathcal{P}_{\mathrm{d} \mathbf{l}} u(x) & =\int_{\partial \mathcal{D}}\left(t(u), n\left(*^{-1} \Phi(x, \cdot)\right)\right)_{y} d s, & & x \in \mathcal{D}^{\prime} \backslash \partial \mathcal{D} ; \\
\mathcal{P}_{\mathrm{v}} f(x) & =\int_{\mathcal{D}}\left(f, *^{-1} \Phi(x, \cdot)\right)_{y} d y, & & x \in \mathcal{D}^{\prime},
\end{aligned}
$$

for $u \in H^{p, \gamma}(\mathcal{D}, E)$ and $f \in H^{0, \gamma-p}(\mathcal{D}, F)$. By the above, the integrals make sense provided $\gamma \geq p$.

Since $\mathcal{P}_{\mathrm{d} \mathbf{l}} u$ does depend only on the Cauchy data $t(u)$ of $u$ on $\partial \mathcal{D}$, the designation $\mathcal{P}_{\mathrm{dl}} u_{0}$ still makes sense for any $u_{0} \in \oplus H^{p-m_{j}-\frac{1}{2}, p-m_{j}-\frac{1}{2}}\left(\partial \mathcal{D}, F_{j}\right)$.

Let $\left(\mathcal{P}_{\mathrm{v}} f\right)^{-}$and $\left(\mathcal{P}_{\mathrm{v}} f\right)^{+}$be the restrictions of $\mathcal{P}_{\mathrm{v}} f$ to $\mathcal{D}$ and $\mathcal{D}^{\prime} \backslash \overline{\mathcal{D}}$, respectively. 
Lemma 5.2 There exists a constant $c>0$ with the property that, for every $f \in H^{0, \gamma-p}(\mathcal{D}, F)$, we have

$$
\begin{aligned}
\left\|\left(\mathcal{P}_{\mathrm{v}} f\right)^{-}\right\|_{H^{p, \gamma}(\mathcal{D}, E)} & \leq c\|f\|_{H^{0, \gamma-p}(\mathcal{D}, F)} \\
\left\|\left(\mathcal{P}_{\mathrm{v}} f\right)^{+}\right\|_{H^{p, \gamma}\left(\mathcal{D}^{\prime} \backslash \overline{\mathcal{D}}, E\right)} & \leq c\|f\|_{H^{0, \gamma-p}(\mathcal{D}, F)}
\end{aligned}
$$

Proof. This follows from the boundedness of edge pseudodifferential operators in the weighted Sobolev spaces, [Sch98].

This lemma gives us a hint that the proper choice of the weight exponent $\gamma$ should be $\gamma=p$.

Lemma 5.3 For every $u \in H^{p, \gamma}(\mathcal{D}, E)$,

$$
\int_{\mathcal{D}}\left(P u, *^{-1} \Phi(x, \cdot)\right)_{y} d y+\int_{\mathcal{D}^{\prime} \backslash \overline{\mathcal{D}}}\left(P \mathcal{E}(u), *^{-1} \Phi(x, \cdot)\right)_{y} d y= \begin{cases}u(x), & x \in \mathcal{D}, \\ \mathcal{E}(u)(x), & x \in \mathcal{D}^{\prime} \backslash \overline{\mathcal{D}} .\end{cases}
$$

Proof. Since $\mathcal{G}(x, y)$ differs from $G(x, y)$ by a kernel $R(x, y)$ which satisfies $\Delta^{\prime}(y, D) R(x, y)=0$ over $\mathcal{O} \times \mathcal{O}$, it follows that

$$
\begin{aligned}
\Phi P & =\mathcal{G} P^{*} P \\
& =(G-R) \Delta \\
& =\mathrm{Id}
\end{aligned}
$$

on compactly supported sections of $\left.E\right|_{\mathcal{O}}$. In other words, $\Phi$ is a left fundamental solution of $P$ on $\mathcal{O}$. By the Green formula,

$$
\begin{aligned}
& -\int_{\partial\left(\mathcal{D}^{\prime} \backslash \overline{\mathcal{D}}\right)}\left(t(\mathcal{E}(u)), n\left(*^{-1} \Phi(x, \cdot)\right)_{y} d s\right. \\
& \quad+\int_{\mathcal{D}^{\prime} \backslash \overline{\mathcal{D}}}\left(P \mathcal{E}(u), *^{-1} \Phi(x, \cdot)\right)_{y} d y= \begin{cases}0, & x \in \mathcal{D}, \\
\mathcal{E}(u)(x), & x \in \mathcal{D}^{\prime} \backslash \overline{\mathcal{D}} .\end{cases}
\end{aligned}
$$

We now make use of the fact that $t(\mathcal{E}(u))=t(u)$ on $\partial \mathcal{D}$ and $t(\mathcal{E}(u))=0$ on $\partial \mathcal{D}^{\prime}$. Hence we deduce that the integral over $\partial\left(\mathcal{D}^{\prime} \backslash \overline{\mathcal{D}}\right)$ on the left hand side is equal to $-\mathcal{P}_{\mathrm{dl}} u(x)$, for all $x \in \mathcal{D}^{\prime} \backslash \partial \mathcal{D}$. This latter potential can in turn be expressed from the Green formula for $u$ over the domain $\mathcal{D}$, thus implying the desired equality.

If $\gamma \geq 1$, then the elements of $H^{p, \gamma}(\mathcal{D}, E)$ vanish on the boundary of $\sigma$, to which refers the weight exponent $\gamma$. Denote by $H_{[\sigma]}^{p, \gamma}(\mathcal{D}, E)$ the closure in $H^{p, \gamma}(\mathcal{D}, E)$ of the $C^{\infty}$ sections of $E$ vanishing near $\bar{\sigma}$. 
Proposition 5.4 Suppose $u \in H^{p, \gamma}(\mathcal{D}, E)$ and $f \in H^{0, \gamma-p}(\mathcal{D}, F)$. For each $v \in H_{[\sigma]}^{p, 2 p-\gamma}(\mathcal{D}, E)$, it follows that

$$
\begin{aligned}
h\left(\mathcal{P}_{\mathrm{v}} f, v\right) & =\int_{\mathcal{D}}(f, P v)_{x} d x \\
h\left(\mathcal{P}_{\mathrm{d} \mathbf{l}} u, v\right) & =\int_{\mathcal{D}^{\prime} \backslash \overline{\mathcal{D}}}(P \mathcal{E}(u), P \mathcal{E}(v))_{x} d x
\end{aligned}
$$

Proof. Indeed, if $f \in H^{p, \gamma-p}(\mathcal{D}, F)$ and $v \in H^{p, 2 p-\gamma}(\mathcal{D}, E)$, then integration by parts gives

$$
\int_{\mathcal{D}}(f, P v)_{x} d x-\int_{D}\left(P^{*} f, v\right)_{x} d x=\int_{\partial \mathcal{D}}(n(f), t(v))_{x} d s
$$

Analogously,

$$
\int_{\mathcal{D}^{\prime} \backslash \mathcal{D}}(P \mathcal{E}(u), P \mathcal{E}(v))_{x} d x=-\int_{\partial \mathcal{D}}(n(P \mathcal{E}(u)), t(v))_{x} d s
$$

for all $u \in H^{2 p, \gamma}(\mathcal{D}, E)$ and $v \in H^{p, 2 p-\gamma}(\mathcal{D}, E)$, because $t(\mathcal{E}(v))=t(v)$ on $\partial \mathcal{D}$ and $t(\mathcal{E}(v))=0$ on $\partial \mathcal{D}^{\prime}$.

Fix $u \in H^{2 p, \gamma}(\mathcal{D}, E)$ and $v \in H^{p, 2 p-\gamma}(\mathcal{D}, E)$, and apply formula (5.2) for $f=P u$. Using (5.2) and (5.3), we get

$$
h(u, v)=\int_{\partial \mathcal{D}}(n(P u)-n(P \mathcal{E}(u)), t(v))_{x} d s+\int_{\mathcal{D}}(\Delta u, v)_{x} d x .
$$

Let us take $f \in C_{\text {comp }}^{\infty}(\mathcal{D}, F)$. In this case integrating by parts shows readily that

$$
\mathcal{P}_{\mathrm{v}} f(x)=\int_{\mathcal{D}}\left(P^{*} f, *_{E}^{-1} \mathcal{G}(x, \cdot)\right)_{y} d y
$$

for all $x \notin \partial \mathcal{D}$. From Proposition 4.3 we deduce that $\mathcal{P}_{\mathrm{v}} f$ is a $C^{\infty}$ section of $E$ over $\mathcal{D}^{\prime} \backslash \bar{\sigma}$, such that $t\left(\mathcal{P}_{\mathrm{v}} f\right)$ vanishes on both $\sigma$ and $\partial \mathcal{D}^{\prime}$. Moreover, it satisfies $\Delta \mathcal{P}_{\mathrm{v}} f=0$ on $\mathcal{D}^{\prime} \backslash \overline{\mathcal{D}}$. Hence it follows that $\mathcal{E}\left(\mathcal{P}_{\mathrm{v}} f\right)^{-}$is simply equal to the restriction of $\mathcal{P}_{\mathrm{v}} f$ to $\mathcal{D}^{\prime} \backslash \overline{\mathcal{D}}$, for $t\left(\mathcal{P}_{\mathrm{v}} f\right)^{+}=t\left(\mathcal{P}_{\mathrm{v}} f\right)^{-}$on $\partial \mathcal{D} \backslash \bar{\sigma}$. Now we can substitute $\left(\mathcal{P}_{\mathrm{v}} f\right)^{-}$for $u$ in the formula above, to obtain

$$
h\left(\mathcal{P}_{\mathrm{v}} f, v\right)=\int_{\partial \mathcal{D}}\left(n\left(P\left(\mathcal{P}_{\mathrm{v}} f\right)^{-}\right)-n\left(P\left(\mathcal{P}_{\mathrm{v}} f\right)^{+}\right), t(v)\right)_{x} d s+\int_{\mathcal{D}}\left(\Delta \mathcal{P}_{\mathrm{v}} f, v\right)_{x} d x
$$

Since $\mathcal{P}_{\mathrm{v}} f$ is smooth away from $\bar{\sigma}$ in $\mathcal{D}^{\prime}$, the jump of $n\left(P \mathcal{P}_{\mathrm{v}} f\right)$ across $\partial \mathcal{D} \backslash \bar{\sigma}$ is zero. On the other hand, this jump has nothing to do on the rest part of the boundary of $\mathcal{D}$, provided that $t(v)=0$ on $\sigma$. Thus, the first summand on the right hand side of the last equality is equal to zero, if $v$ varies over 
$H_{[\sigma]}^{p, 2 p-\gamma}(\mathcal{D}, E)$. Taking into account that $\Delta \mathcal{P}_{\mathrm{v}} f(x)=P^{*} f(x)$ for all $x \in \mathcal{D}$, we get

$$
\begin{aligned}
h\left(\mathcal{P}_{\mathrm{v}} f, v\right) & =\int_{\mathcal{D}}\left(P^{*} f, v\right)_{x} d x \\
& =\int_{\mathcal{D}}(f, P v)_{x} d x .
\end{aligned}
$$

Since $C_{\text {comp }}^{\infty}(\mathcal{D}, F)$ is dense in $H^{0, \gamma-p}(\mathcal{D}, F)$, this formula holds for every $f \in H^{0, \gamma-p}(\mathcal{D}, F)$ and $v \in H_{[\sigma]}^{p, 2 p-\gamma}(\mathcal{D}, E)$, as desired.

Finally, we can express the double layer potential $\mathcal{P}_{\mathrm{dl}} u$ from the Green formula for $P$ in $\mathcal{D}$ as $\mathcal{P}_{\mathrm{dl}} u=u-\mathcal{P}_{\mathrm{v}} P u$. Hence

$$
\begin{aligned}
h\left(\mathcal{P}_{\mathrm{dl}} u, v\right) & =h(u, v)-h\left(\mathcal{P}_{\mathrm{v}} P u, v\right) \\
& =\int_{\mathcal{D}^{\prime} \backslash \overline{\mathcal{D}}}(P \mathcal{E}(u), P \mathcal{E}(v))_{x} d x
\end{aligned}
$$

for any $u \in H^{p, \gamma}(\mathcal{D}, u)$ and $v \in H_{[\sigma]}^{p, 2 p-\gamma}(\mathcal{D}, E)$, as is clear from what has already been proved. This establishes the formula.

Not only does Proposition 5.4 specify the adjoint operator for $P$ under the scalar product $h(u, v)$, but does also show the self-adjointness of the double layer potential.

Lemma 5.5 The mappings $t_{-}, t_{+}$induce topological isomorphisms of Hilbert spaces, namely

$$
\begin{aligned}
& H^{p, \gamma}(\mathcal{D}, E) \cap \mathcal{S}_{\Delta}(\mathcal{D}) \stackrel{t_{-}}{\longrightarrow} \oplus H^{p-m_{j}-\frac{1}{2}, \gamma-m_{j}-\frac{1}{2}}\left(\partial \mathcal{D}, F_{j}\right), \\
& H^{p, \gamma}\left(\mathcal{D}^{\prime} \backslash \overline{\mathcal{D}}, E\right) \cap \mathcal{S}_{\Delta}\left(\hat{\mathcal{D}}^{\prime} \backslash \overline{\mathcal{D}}\right) \stackrel{t_{+}}{\longrightarrow} \oplus H^{p-m_{j}-\frac{1}{2}, \gamma-m_{j}-\frac{1}{2}}\left(\partial \mathcal{D}, F_{j}\right) .
\end{aligned}
$$

Proof. This follows from Lemma 4.2 as is explained at the very beginning of this section.

Proposition 5.6 The topologies induced in $H^{p, p}(\mathcal{D}, E)$ by $h(\cdot, \cdot)$ and by the original scalar product are equivalent.

Proof. As the mapping (5.1) is continuous, we see, for any $u \in H^{p, p}(\mathcal{D}, E)$, that

$$
\begin{aligned}
h(u, u) & =\|P u\|_{L^{2}(\mathcal{D}, F)}^{2}+\|P \mathcal{E}(u)\|_{L^{2}\left(\mathcal{D}^{\prime} \backslash \overline{\mathcal{D}}, F\right)}^{2} \\
& \leq c\left(\|u\|_{H^{p, p}(\mathcal{D}, E)}^{2}+\|\mathcal{E}(u)\|_{H^{p, p}\left(\mathcal{D}^{\prime} \backslash \overline{\mathcal{D}}, E\right)}^{2}\right) \\
& \leq c\left(1+c^{\prime}\right)\|u\|_{H^{p, p}(\mathcal{D}, E)}^{2},
\end{aligned}
$$


the constants $c$ and $c^{\prime}$ being independent of $u$. Here, we use the identification $L^{2}(\mathcal{D}, F)=H^{0,0}(\mathcal{D}, F)$ and the fact that $P$ acts continuously from $H^{s, \gamma}(\mathcal{D}, E)$ to $H^{s-p, \gamma-p}(\mathcal{D}, F)$.

Conversely, Lemmas 5.2 and 5.3 imply that

$$
\begin{aligned}
\frac{1}{2}\|u\|_{H^{p, p}(\mathcal{D}, E)}^{2} & \leq\left\|\mathcal{P}_{\mathbf{v}} P u\right\|_{H^{p, p}(\mathcal{D}, E)}^{2}+\left\|\mathcal{P}_{\mathbf{v}} P \mathcal{E}(u)\right\|_{H^{p, p}(\mathcal{D}, E)}^{2} \\
& \leq c\|P u\|_{L^{2}(\mathcal{D}, F)}^{2}+c\|P \mathcal{E}(u)\|_{L^{2}\left(\mathcal{D}^{\prime} \backslash \overline{\mathcal{D}}, F\right)}^{2} \\
& =c h(u, u),
\end{aligned}
$$

with $c$ a constant independent of $u$. This completes the proof.

As mentioned above, Proposition 5.4 allows one to specify the adjoint operator of $P$ with respect to the scalar product $h(u, v)$ on $H_{[\sigma]}^{p, p}(\mathcal{D}, E)$ as the volume potential $\mathcal{P}_{\mathrm{v}}$.

Theorem 5.7 In the Hilbert space $H_{[\sigma]}^{p, p}(\mathcal{D}, E)$ endowed with the scalar product $h(\cdot, \cdot)$, we have $\|P\| \leq 1$ and $P^{*}=\mathcal{P}_{\mathrm{v}}$.

Proof. This is an immediate consequence of Propositions 5.1, 5.4 and 5.6.

\section{The case of constant coefficients}

Note that we may use similar arguments for the case where $P$ is a first order elliptic homogeneous differential operator with constant coefficients in $X=\mathbb{R}^{n}$, $n \geq 2$, and $\mathcal{D}^{\prime}$ is taken to be the whole $X$. To this end, however, we need to correct the scalar product $h(\cdot, \cdot)$.

As $\Delta=P^{*} P$ is a second order elliptic homogeneous differential operator with constant coefficients, it has a fundamental solution of convolution type

$$
G(x-y)=G_{1}\left(\frac{x-y}{|x-y|}\right)|x-y|^{2-n}+G_{2}(x-y) \log |x-y|,
$$

where $G_{1}(z)$ is a $(k \times k)$-matrix of real analytic functions near the unit sphere in $\mathbb{R}^{n}$, and $G_{2}(z)$ is a $(k \times k)$-matrix of homogeneous polynomials of degree $2-n$. In particular, $G_{2}(z) \equiv 0$ for $n>2$.

For example, if $P$ is the gradient operator in $\mathbb{R}^{n}$, then $P^{*} P$ is the -1 multiple of the Laplace operator in $\mathbb{R}^{n}$. If $P$ is the Cauchy-Riemann system in $\mathbb{C}^{N}\left(\cong \mathbb{R}^{2 N}\right)$, then $P^{*} P$ is the $-\frac{1}{4}$ multiple of the Laplace operator in $\mathbb{R}^{2 N}$. These examples extend to various matrix factorisations of the Laplace operator in $\mathbb{R}^{n}$.

Let $K$ be a compact set in $\mathbb{R}^{n}$. Denote by $\mathcal{S}_{\Delta}\left(\hat{\mathbb{R}}^{n} \backslash K\right)$ the set of all functions $u$ on $\mathbb{R}^{n} \backslash K$ with values in $\mathbb{C}^{k}$, such that 
1) $\Delta u=0$ in $\mathbb{R}^{n} \backslash K$;

2) there exists a limit $\lim _{|x| \rightarrow \infty} u(x)=0$, for $n>2$, or $\lim _{|x| \rightarrow \infty} u(x) \in \mathbb{C}^{k}$, for $n=2$.

Let moreover $\mathcal{S}_{P}\left(\hat{\mathbb{R}}^{n} \backslash K\right)$ be the closed subspace of $\mathcal{S}_{\Delta}\left(\hat{\mathbb{R}}^{n} \backslash K\right)$ consisting of those functions $\mathcal{S}_{P}\left(\mathbb{R}^{n} \backslash K\right)$ which satisfy $\lim _{|x| \rightarrow \infty} u(x)=0$.

Fix a point $x^{0}$ in the domain $\mathcal{D}$. Then Theorem 3.2.15 from [Tar95] implies that every function $u \in \mathcal{S}_{\Delta}\left(\hat{\mathbb{R}}^{n} \backslash K\right)$ can be expanded into a Laurent series

$$
u(x)=c\left(u, x^{0}\right)+\sum_{\alpha \in \mathbb{Z}_{+}^{n}} D^{\alpha} G\left(x-x^{0}\right) c_{\alpha}\left(u, x^{0}\right)
$$

in the complement of a closed ball $B$ with centre $x^{0}$ and a radius $R$ depending on $P$ and $K$. The series converges absolutely and uniformly in $\mathbb{R}^{n} \backslash B$, and the coefficients $c\left(u, x^{0}\right)$ and $c_{\alpha}\left(u, x^{0}\right)$ are uniquely defined. In fact, $c\left(u, x^{0}\right)=0$, for $n>2$, and $c_{0}\left(u, x^{0}\right)=0$, for $n=2$.

Mention that the conditions at the point at infinity imposed on the elements of $\mathcal{S}_{\Delta}\left(\hat{\mathbb{R}}^{n} \backslash K\right)$ are necessary for the well-posedness of the Dirichlet problem for $\Delta$ in a domain with compact complement. Thus, instead of Lemma 4.2 we need the following one.

Lemma 6.1 Let $n>2$ and $\gamma$ be close to 1 . For each $u_{0} \in H^{s-\frac{1}{2}, \gamma-\frac{1}{2}}(\sigma, E)$, there exists a unique function $u \in H^{s, \gamma}\left(\mathbb{R}^{n} \backslash \bar{\sigma}, E\right) \cap \mathcal{S}_{\Delta}\left(\hat{\mathbb{R}}^{n} \backslash \bar{\sigma}\right)$ satisfying $u=u_{0}$ on $\sigma$.

Proof. The proof is just in the same framework as that of Lemma 4.2 because the ellipticity at the point at infinity is fulfilled.

The lemma is still true for $n=2$ if we slightly modify the formulation by requiring $u \in H^{s, \gamma}(B \backslash \bar{\sigma}, E)$, for each ball $B$ containing $\bar{\sigma}$.

Since the Dirichlet problem for $\Delta$ in $\mathbb{R}^{n} \backslash \bar{\sigma}$ is elliptic, the solution $u$ can be represented by

$$
u(x)=\int_{\sigma}\left(u, n\left(P *_{E}^{-1} \mathcal{G}(x, \cdot)\right)^{+}-n\left(P *_{E}^{-1} \mathcal{G}(x, \cdot)\right)^{-}\right)_{y} d s,
$$

where $\mathcal{G}(x, y)$ is what is usually called the Green function of the problem and $n\left(P *^{-1} \mathcal{G}(x, \cdot)\right)^{ \pm}$are the limit values of $n\left(P *^{-1} \mathcal{G}(x, \cdot)\right)$ on $\sigma$ from $\mathbb{R}^{n} \backslash \overline{\mathcal{D}}$ and $\mathcal{D}$, respectively.

Proposition 6.2 There is a Green function $\mathcal{G}(x, y)$ of the Dirichlet problem for $\Delta$ in $\mathbb{R}^{n} \backslash \bar{\sigma}$, i.e., a two-sided fundamental solution of $\Delta$ in $\mathbb{R}^{n} \backslash \bar{\sigma}$, such that 
1) $\mathcal{G}$ extends to a smooth matrix-valued function away from the diagonal in $\left(\mathbb{R}^{n} \backslash \partial \sigma\right) \times\left(\mathbb{R}^{n} \backslash \partial \sigma\right)$, the smoothness near $\sigma \times \sigma$ being understood sidewise;

2) $\mathcal{G}(\cdot, y)$ vanishes on $\sigma$ and the difference $G(\cdot, y)-\mathcal{G}(\cdot, y)$ behaves at the point at infinity as an element of $\mathcal{S}_{\Delta}\left(\hat{\mathbb{R}}^{n} \backslash \bar{\sigma}\right)$, for each $y \in \mathbb{R}^{n} \backslash \bar{\sigma}$.

Proof. In view of Lemma 6.1, this is a very particular case of Proposition 4.3.

Solving the Dirichlet problem for $\Delta$ in $\mathbb{R}^{n} \backslash \overline{\mathcal{D}}$ instead of $\mathbb{R}^{n} \backslash \bar{\sigma}$, we get a linear isomorphism

$$
H^{1, \gamma}(B \backslash \overline{\mathcal{D}}, E) \cap \mathcal{S}_{\Delta}\left(\hat{\mathbb{R}}^{n} \backslash \overline{\mathcal{D}}\right) \stackrel{t_{+}}{\longrightarrow} H^{\frac{1}{2}, \gamma-\frac{1}{2}}(\partial \mathcal{D}, E)
$$

by $\left.u \mapsto u\right|_{\partial \mathcal{D}}$, where $B$ is any ball containing $\overline{\mathcal{D}}$ Then, composing the inverse $t_{+}^{-1}$ with the trace operator

$$
H^{1, \gamma}(\mathcal{D}, E) \stackrel{t_{-}}{\longrightarrow} H^{\frac{1}{2}, \gamma-\frac{1}{2}}(\partial \mathcal{D}, E)
$$

we arrive at a continuous linear mapping

$$
H^{1, \gamma}(\mathcal{D}, E) \stackrel{\mathcal{E}}{\longrightarrow} H^{1, \gamma}(B \backslash \overline{\mathcal{D}}, E) \cap \mathcal{S}_{\Delta}\left(\hat{\mathbb{R}}^{n} \backslash \overline{\mathcal{D}}\right)
$$

(cf. (5.1)).

Theorem 6.3 The Hermitian form

$$
h(u, v)=\int_{\mathcal{D}}(P u, P v)_{y} d y+\int_{\mathbb{R}^{n} \backslash \mathcal{D}}(P \mathcal{E}(u), P \mathcal{E}(v))_{y} d y+(\mathcal{E}(u)(\infty), \mathcal{E}(v)(\infty))
$$

is a scalar product on the space $H^{1,1}(\mathcal{D}, E)$ inducing the topology equivalent to the original one.

Proof. This follows by the same method as in the proof Proposition 5.6. The main ingredient thereof is Lemma 5.3 which still remains valid in the case $\mathcal{D}^{\prime}=\mathbb{R}^{n}$.

For $u \in H^{1, \gamma}(\mathcal{D}, E)$, we introduce the double layer potential $\mathcal{P}_{\mathrm{dl}} u$ and, for $f \in H^{0, \gamma-1}(\mathcal{D}, F)$, the volume potential $\mathcal{P}_{\mathrm{v}} f$ on $\mathbb{R}^{n} \backslash \partial \mathcal{D}$ by the same formulas as in Section 5 .

Proposition 6.4 Suppose $u \in H^{1, \gamma}(\mathcal{D}, E)$ and $f \in H^{0, \gamma-1}(\mathcal{D}, F)$. For each $v \in H_{[\sigma]}^{1,2-\gamma}(\mathcal{D}, E)$, it follows that

$$
\begin{aligned}
h\left(\mathcal{P}_{\mathrm{v}} f, v\right) & =\int_{\mathcal{D}}(f, P v)_{x} d x, \\
h\left(\mathcal{P}_{\mathrm{dl}} u, v\right) & =\int_{\mathbb{R}^{n} \backslash \overline{\mathcal{D}}}(P \mathcal{E}(u), P \mathcal{E}(v))_{x} d x+(\mathcal{E}(u)(\infty), \mathcal{E}(v)(\infty)) .
\end{aligned}
$$

Proof. The proof is similar to the proof of Proposition 5.4. 


\section{Iterations}

The following theorem can actually be formulated in the abstract context of selfadjoint operators in Hilbert spaces, cf. Theorem 1.2.

Theorem 7.1 In the strong operator topology on the space $\mathcal{L}\left(H_{[\sigma]}^{p, p}(\mathcal{D}, E)\right)$, we have

$$
\begin{aligned}
\lim _{N \rightarrow \infty} \mathcal{P}_{\mathrm{dl}}^{N} & =\pi_{V_{1}}, \\
\lim _{N \rightarrow \infty}\left(\mathcal{\mathcal { P }}_{\mathbf{v}} P\right)^{N} & =\pi_{V_{0}},
\end{aligned}
$$

where

$$
\begin{aligned}
& V_{0}=\left\{u \in H_{[\sigma]}^{p, p}(\mathcal{D}, E): P \mathcal{E}(u)=0 \text { in } \mathcal{D}^{\prime} \backslash \overline{\mathcal{D}}\right\} \\
& V_{1}=\left\{u \in H_{[\sigma]}^{p, p}(\mathcal{D}, E): P u=0 \text { in } \mathcal{D}\right\} .
\end{aligned}
$$

Proof. First, Theorem 5.7 implies that the integrals $\mathcal{P}_{\mathrm{dl}}$ and $\mathcal{P}_{\mathrm{v}} P$ define bounded selfadjoint non-negative operators in the Hilbert space $H_{[\sigma]}^{p, p}(\mathcal{D}, E)$ with the scalar product $h(\cdot, \cdot)$. Moreover, their norms do not exceed 1 .

By the spectral theorem for bounded selfadjoint operators we conclude readily that

$$
\mathcal{P}_{\mathrm{dl}}^{N}=\int_{0-}^{1+0} \lambda^{N} d I(\lambda)
$$

where $(I(\lambda))_{0 \leq \lambda \leq 1}$ is a resolution of the identity in the Hilbert space $H_{[\sigma]}^{p, p}(\mathcal{D}, E)$ corresponding to the selfadjoint operator $0 \leq \mathcal{P}_{\mathrm{dl}} \leq I$ (see, for instance, Sections 5, 6 in [Yos65, Ch. XI]).

Passing to the limit in (7.1) yields

$$
\lim _{N \rightarrow \infty} \mathcal{P}_{\mathrm{dl}}^{N}=I(1+0)-I(1-0),
$$

the operator on the right side being an orthogonal projection of $H_{[\sigma]}^{p, p}(\mathcal{D}, E)$ onto a (closed) subspace $V(1)$. Obviously,

$$
\left(I-\mathcal{P}_{\mathrm{dl}}\right) \lim _{N \rightarrow 0} \mathcal{P}_{\mathrm{dl}}^{N} u=0
$$

for all $u \in H_{[\sigma]}^{p, p}(\mathcal{D}, E)$, i.e., $V(1) \subset \operatorname{ker}\left(I-\mathcal{P}_{\mathrm{dl}}\right)$. Finally, if $u \in \operatorname{ker}\left(I-\mathcal{P}_{\mathrm{dl}}\right)$ then

$$
\begin{aligned}
u & =\mathcal{P}_{\mathrm{dl}} u+\left(I-\mathcal{P}_{\mathrm{dl}}\right) u \\
& =\mathcal{P}_{\mathrm{dl}} u \\
& =\mathcal{P}_{\mathrm{dl}}^{N} u
\end{aligned}
$$

for every $N \geq 0$. Hence it follows that

$$
u=\lim _{N \rightarrow \infty} \mathcal{P}_{\mathrm{dl}}^{N} u,
$$


and so $V(1)=\operatorname{ker}\left(I-\mathcal{P}_{\mathrm{dl}}\right)$ whence

$$
\lim _{N \rightarrow \infty} \mathcal{P}_{\mathrm{dl}}^{N}=\pi_{\mathrm{ker}} \mathcal{P}_{\mathrm{v}} P .
$$

Arguing in the same way we obtain

$$
\lim _{N \rightarrow \infty}\left(\mathcal{P}_{\mathrm{v}} P\right)^{N}=\pi_{\mathrm{ker}} \mathcal{P}_{\mathrm{dl}} .
$$

Now Proposition 5.4 implies that the null-space of $\mathcal{P}_{\mathrm{v}} P$ in $H_{[\sigma]}^{p, p}(\mathcal{D}, E)$ coincides with $H_{[\sigma]}^{p, p}(\mathcal{D}, E) \cap \mathcal{S}_{P}(\mathcal{D})$.

Finally, Proposition 5.4 and Lemma 5.3, if combined with the Green formula for $P$, imply that $\mathcal{P}_{\mathrm{dl}} u=0$ if and only if $P \mathcal{E}(u)=0$ in $\mathcal{D}^{\prime} \backslash \overline{\mathcal{D}}$.

Recall that the operator $P$ bears the uniqueness property for the Cauchy problem in the small on $X$. Hence, if the boundary of $\mathcal{D}$ is connected, Theorem 10.3.5 of [Tar95] implies that $V_{0}$ coincides with the space of all $u \in H^{p, p}(\mathcal{D}, E)$ such that $t(u)=0$ on $\partial \mathcal{D}$.

On the other hand, if $\sigma \neq \emptyset$, then the uniqueness theorem for $P$ gives $V_{1} \equiv 0$.

Corollary 7.2 In the strong operator topology of the space $\mathcal{L}\left(H_{[\sigma]}^{p, p}(\mathcal{D}, E)\right)$, we have

$$
\begin{aligned}
I & =\lim _{N \rightarrow \infty} \mathcal{P}_{\mathrm{dl}}^{N}+\sum_{\nu=0}^{\infty} \mathcal{P}_{\mathrm{dl}}^{\nu} \mathcal{P}_{\mathrm{v}} P \\
I & =\lim _{N \rightarrow \infty}\left(\mathcal{P}_{\mathrm{v}} P\right)^{N}+\sum_{\nu=0}^{\infty}\left(\mathcal{P}_{\mathrm{v}} P\right)^{\nu} \mathcal{P}_{\mathrm{dl}} .
\end{aligned}
$$

Proof. Indeed, the Green formula $\mathcal{P}_{\mathrm{dl}}+\mathcal{P}_{\mathrm{v}} P=I$ in $\mathcal{D}$ implies

$$
\begin{aligned}
I & =\mathcal{P}_{\mathrm{dl}}^{N}+\sum_{\nu=0}^{N-1} \mathcal{P}_{\mathrm{dl}}^{\nu} \mathcal{P}_{\mathrm{v}} P \\
& =\left(\mathcal{P}_{\mathrm{v}} P\right)^{N}+\sum_{\nu=0}^{N-1}\left(\mathcal{P}_{\mathrm{v}} P\right)^{\nu} \mathcal{P}_{\mathrm{dl}},
\end{aligned}
$$

for every $N \in \mathbb{N}$. Now using Theorem 7.1 we can pass to the limit for $N \rightarrow \infty$, thus obtaining (7.3) and (7.2).

For applications to the Cauchy problem for solutions of $P u=0$ in $\mathcal{D}$ with data on $\sigma$, we also need the following result.

Corollary 7.3 Suppose $\sigma \neq \emptyset$. Then, in the strong operator topology of $\mathcal{L}\left(L^{2}(\mathcal{D}, F)\right)$, we have

$$
I=\pi_{\mathrm{ker}} \mathcal{P}_{\mathrm{v}}+\sum_{\nu=0}^{\infty} P \mathcal{P}_{\mathrm{dl}}^{\nu} \mathcal{P}_{\mathrm{v}}
$$


Proof. Arguing as in the proof of Corollary 7.2, with Theorem 7.1 replaced by an abstract Theorem 2.2 of [Sh199], we obtain

$$
\begin{aligned}
I & =\lim _{N \rightarrow \infty}\left(I-P \mathcal{P}_{\mathrm{v}}\right)^{N}+\sum_{\nu=0}^{\infty}\left(I-P \mathcal{P}_{\mathrm{v}}\right)^{\nu} P \mathcal{P}_{\mathrm{v}} \\
& =\pi_{\mathrm{ker} P \mathcal{P}_{\mathrm{v}}}+\sum_{\nu=0}^{\infty}\left(I-P \mathcal{P}_{\mathrm{v}}\right)^{\nu} P \mathcal{P}_{\mathrm{v}}
\end{aligned}
$$

$I$ being the identity operator on $L^{2}(\mathcal{D}, F)$.

Assume that $f \in L^{2}(\mathcal{D}, F)$ and $P \mathcal{P}_{\mathrm{v}} f=0$ in $\mathcal{D}$. As $t\left(\mathcal{P}_{\mathrm{v}} f\right)=0$ on $\sigma$, it follows, by the uniqueness property for the Cauchy problem in the small, that $\mathcal{P}_{\mathrm{v}} f=0$ in $\mathcal{D}$. Consequently, the kernel of $P \mathcal{P}_{\mathrm{v}}$ on $L^{2}(\mathcal{D}, F)$ coincides with that of $\mathcal{P}_{\mathrm{v}}$.

To complete the proof, it suffices to observe that

$$
\begin{aligned}
\left(I-P \mathcal{P}_{\mathrm{v}}\right)^{\nu} P \mathcal{P}_{\mathrm{v}} & =P\left(I-\mathcal{P}_{\mathrm{v}} P\right)^{\nu} \mathcal{P}_{\mathrm{v}} \\
& =P \mathcal{P}_{\mathrm{dl}}^{\nu} \mathcal{P}_{\mathrm{v}}
\end{aligned}
$$

for any $\nu \in \mathbb{Z}_{+}$, which establishes the formula.

\section{Cauchy problem}

Consider the following Cauchy problem, for the operator $P$ and the Dirichlet system $\left\{B_{j}\right\}_{j=0}^{p-1}$. Given $f \in L^{2}(\mathcal{D}, F)$ and $u_{0} \in \oplus H^{p-m_{j}-\frac{1}{2}, p-m_{j}-\frac{1}{2}}\left(\sigma, F_{j}\right)$, find a section $u \in H^{p, p}(\mathcal{D}, E)$ satisfying

$$
\left\{\begin{aligned}
P u & =f \quad \text { in } \quad \mathcal{D}, \\
t(u) & =u_{0} \text { on } \quad \sigma .
\end{aligned}\right.
$$

This problem is well-known to be ill-posed unless $\sigma=\partial \mathcal{D}$. Using Corollary 7.2 we obtain easily approximate solutions to the problem. To this end, set

$$
\mathcal{P}_{\mathrm{dl} l} u_{0}(x)=-\int_{\sigma}\left(u_{0}, n\left(*^{-1} \Phi(x, \cdot)\right)\right)_{y} d s, \quad x \in \mathcal{D} .
$$

Note that the integral on the right hand side of formula (8.2) is well-defined because of the properties of $\Phi(x, \cdot)$ and $u_{0}$.

Theorem 8.1 The problem (8.1) has at most one solution. It is solvable if and only if $f \perp \operatorname{ker} \mathcal{P}_{\mathrm{v}}$ and the series

$$
u=\mathcal{P}_{\mathrm{d} \mathbf{l}} u_{0}+\sum_{\nu=0}^{\infty} \mathcal{P}_{\mathrm{dl}}^{\nu} \mathcal{P}_{\mathbf{v}}\left(f-P \mathcal{P}_{\mathrm{d} \mathbf{l}} u_{0}\right)
$$


converges in $H^{p, p}(\mathcal{D}, E)$. Moreover, the series, if converges, is the solution to this problem.

Proof. For the uniqueness theorem for solutions of (8.1) we refer the reader to $[$ Tar95, 10.3.1].

Lemma 4.2 and Proposition 4.3 imply that the potential $\mathcal{P}_{\mathrm{dl}} u_{0}$ belongs to $H^{p, p}(\mathcal{D}, E) \cap \mathcal{S}_{\Delta}(\mathcal{D})$ and satisfies $t\left(\mathcal{P}_{\mathrm{dl}} u_{0}\right)=u_{0}$ on $\sigma$. Thus, by setting $u=\mathcal{P}_{\mathrm{dl}} u_{0}+\tilde{u}$ we reduce the problem (8.1) to the problem of finding a function $\tilde{u} \in H^{p, p}(\mathcal{D}, E)$ such that

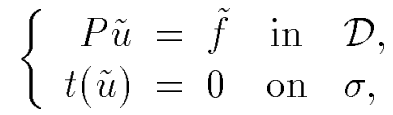

where $\tilde{f}=f-P \mathcal{P}_{\mathrm{d} l} u_{0}$. It is a simple matter to see, by Theorem 5.7, that $\tilde{f} \perp \operatorname{ker} \mathcal{P}_{\mathrm{v}}$ if and only if $f \perp \operatorname{ker} \mathcal{P}_{\mathrm{v}}$.

Let the problem (8.4) be solvable. Then $\tilde{f} \perp \operatorname{ker} \mathcal{P}_{\mathrm{v}}$. Moreover, equality (7.2) implies that the series $\tilde{u}=\sum_{\nu=0}^{\infty} \mathcal{P}_{\mathrm{dl}}^{\nu} \mathcal{P}_{\mathrm{v}} \tilde{f}$ converges and gives a solution to this problem. Therefore, the problem (8.1) is solvable, too, and its solution is presented by (8.3).

Conversely, let $\tilde{f} \perp \operatorname{ker} \mathcal{P}_{\mathrm{v}}$ and the series $(8.3)$ converge in $H^{p, p}(\mathcal{D}, E)$. Then Corollary 7.3 implies that

$$
\begin{aligned}
P \tilde{u} & =\sum_{\nu=0}^{\infty} P \mathcal{P}_{\mathrm{dl}}^{\nu} \mathcal{P}_{\mathrm{v}} \tilde{f} \\
& =\tilde{f}-\pi_{\mathrm{ker}} \mathcal{P}_{\mathrm{v}} \tilde{f} \\
& =\tilde{f}
\end{aligned}
$$

as desired.

The theorem gains in interest if we realise that the null-space of $\mathcal{P}_{\mathrm{v}}$ consists of all $g \in L^{2}(\mathcal{D}, F)$ satisfying $P^{*} g=0$ in $\mathcal{D}$ and $n(g)=0$ on $\partial \mathcal{D}$ (cf. Lemma 10.2.20 in [Tar95]). Hence, $f \perp \operatorname{ker} \mathcal{P}_{\mathrm{v}}$ implies, in particular, that $P_{1} f=0$, for each differential operator $P_{1}$ over $\mathcal{D}$ satisfying $P_{1} P=0$.

\section{Zaremba problem}

Consider the following generalised Zaremba problem. Given $f \in H^{-p,-p}(\mathcal{D}, E)$ and

$$
\begin{aligned}
& u_{0} \in \oplus H^{p-m_{j}-\frac{1}{2}, p-m_{j}-\frac{1}{2}}\left(\sigma, F_{j}\right), \\
& u_{1} \in \oplus H^{-p+m_{j}+\frac{1}{2},-p+m_{j}+\frac{1}{2}}\left(\partial \mathcal{D} \backslash \bar{\sigma}, F_{j}\right),
\end{aligned}
$$

find a section $u \in H^{p, p}(\mathcal{D}, E)$ such that

$$
\left\{\begin{aligned}
\Delta u & =f & \text { in } & \mathcal{D}, \\
t(u) & =u_{0} & \text { on } & \sigma, \\
n(P u) & =u_{1} & \text { on } & \partial \mathcal{D} \backslash \bar{\sigma} .
\end{aligned}\right.
$$


It is worth pointing out that the trace of $n(P u)$ on $\partial \mathcal{D} \backslash \bar{\sigma}$ is not defined for any $u \in H^{p, p}(\mathcal{D}, E)$, for the order of $n \circ P$ is $2 p-1$. To cope with this, a familiar way is to assign an operator $L$ with a dense domain $\operatorname{Dom} L \hookrightarrow H^{p, p}(\mathcal{D}, E)$ to (9.1), such that both $t(u)$ and $n(P u)$ are well-defined for all $u \in$ Dom $L$. More precisely, Dom $L$ is defined to be the completion of $C_{\text {comp }}^{\infty}(\overline{\mathcal{D}} \backslash \partial \sigma, E)$ with respect to the graph norm of $u \mapsto(u, t(u), n(P u))$ in $H^{p, p}(\mathcal{D}, E) \oplus \mathfrak{D} \oplus \mathfrak{N}$, where

$$
\begin{aligned}
& \mathfrak{D}=\oplus H^{p-m_{j}-\frac{1}{2}, p-m_{j}-\frac{1}{2}}\left(\sigma, F_{j}\right), \\
& \mathfrak{N}=\oplus H^{-p+m_{j}+\frac{1}{2},-p+m_{j}+\frac{1}{2}}\left(\partial \mathcal{D} \backslash \bar{\sigma}, F_{j}\right) .
\end{aligned}
$$

For more details, see Roitberg [Roi96] and elsewhere. Then, (9.1) defines a continuous operator $\operatorname{Dom} L \rightarrow H^{-p,-p}(\mathcal{D}, E) \oplus \mathfrak{D} \oplus \mathfrak{N}$ by $L u=(\Delta u, t(u), n(P u))$.

If $P$ is the gradient operator in $\mathbb{R}^{n}$, then (9.1) is just the classical Zaremba problem in $\mathcal{D}$.

As the Dirichlet problem is elliptic, it is easy to reduce the mixed problem (9.1) to that with $u_{0}=0$. To this end, set $u=\mathcal{P}_{\mathrm{dl}} u_{0}+\tilde{u}$, the integral $\mathcal{P}_{\mathrm{dl}} u_{0}$ being given by (8.2). Then the data in (9.1) transform to $\tilde{f}=f$ and $\tilde{u}_{0}=0$, $\tilde{u}_{1}=u_{1}-n\left(P \mathcal{P}_{\mathrm{dl}} u_{0}\right)$.

The advantage of studying the problem (9.1) with $u_{0}=0$ lies in the fact that it can be thought of in the following variational sense. For any $f$ and $u_{1}$, as above, find a $u \in H_{[\sigma]}^{p, p}(\mathcal{D}, E)$ such that

$$
\int_{\mathcal{D}}(P u, P v)_{x} d x=\int_{\mathcal{D}}(f, v)_{x} d x+\int_{\partial \mathcal{D}}\left(u_{1}, t(v)\right)_{x} d s
$$

for all $v \in H_{[\sigma]}^{p, p}(\mathcal{D}, E)$.

For Zaremba data $f$ and $u_{1}$, we introduce the simple layer and volume potentials by

$$
\begin{aligned}
\mathcal{P}_{\mathrm{sl}} u_{1}(x) & =\int_{\partial \mathcal{D} \backslash \bar{\sigma}}\left(u_{1}, t\left(*^{-1} \mathcal{G}(x, \cdot)\right)\right)_{y} d s, & & x \in \mathcal{D} ; \\
\mathcal{G}\left(\chi_{\mathcal{D}} f\right)(x) & =\int_{\mathcal{D}}\left(f, *^{-1} \mathcal{G}(x, \cdot)\right)_{y} d y, & & x \in \mathcal{D},
\end{aligned}
$$

where $\chi_{\mathcal{D}}$ is the characteristic function of $\mathcal{D}$.

Recall, cf. Remark 4.4, that $t\left(*^{-1} \mathcal{G}(x, \cdot)\right)$ vanishes on $\sigma$, for each fixed $x \in \mathcal{D}^{\prime} \backslash \bar{\sigma}$, hence the first integral in (9.3) is actually over all of $\partial \mathcal{D}$.

Theorem 9.1 If $\sigma \neq \emptyset$, then the problem (9.1) has no more than one solution. It is solvable if and only if the series

$$
u=\mathcal{P}_{\mathrm{dl}} u_{0}+\sum_{\nu=0}^{\infty} \mathcal{P}_{\mathrm{dl}}^{\nu}\left(\mathcal{G}\left(\chi_{\mathcal{D}} f\right)+\mathcal{P}_{\mathrm{sl}}\left(u_{1}-n\left(P \mathcal{P}_{\mathrm{dl}} u_{0}\right)\right)\right)
$$

converges in $H^{p, p}(\mathcal{D}, E)$. Moreover, the series, when converges, is the solution to this problem. 
Proof. The uniqueness theorem for the problem (9.1) follows from the variational setting $(9.2)$ immediately. Indeed, let $u \in H_{[\sigma]}^{p, p}(\mathcal{D}, E)$ be any solution of the problem corresponding to the zero data. Then $\int_{\mathcal{D}}(P u, P v)_{x} d x=0$ for all $v \in H_{[\sigma]}^{p, p}(\mathcal{D}, E)$. In particular, substituting $v=u$ we conclude that $u \in H_{[\sigma]}^{p, p}(\mathcal{D}, E) \cap \mathcal{S}_{P}(\mathcal{D})$. Since $P$ bears the uniqueness property for the Cauchy problem in the small on $X$ we see that $u \equiv 0$, which is our claim.

Since $t\left(*^{-1} \mathcal{G}(x, \cdot)\right)=0$ on $\sigma$, for each $x \in \mathcal{D}$, it follows from the Green formula for $P$ that

$$
\mathcal{P}_{\mathrm{v}} P u=\mathcal{G}\left(\chi_{\mathcal{D}} \Delta u\right)+\mathcal{P}_{\mathrm{sl}} n(P u)
$$

for all $u \in H^{p, p}(\mathcal{D}, E)$. Hence, formula $(7.2)$ shows that any solution $\tilde{u}$ to the problem $(9.1)$ with $\tilde{u}_{0}=0$ can be presented by the formula

$$
\tilde{u}=\sum_{\nu=0}^{\infty} \mathcal{P}_{\mathrm{dl}}^{\nu}\left(\mathcal{G}\left(\chi_{\mathcal{D}} f\right)+\mathcal{P}_{\mathrm{sl}} \tilde{u}_{1}\right)
$$

It follows that any solution to the general problem (9.1) can be written by formula (9.4).

Conversely, let the series (9.4) converge to a function $u \in H^{p, p}(\mathcal{D}, E)$. Then the series $(9.5)$ converges, too, to the function $\tilde{u}=u-\mathcal{P}_{\mathrm{dl}} u_{0}$ lying in $H_{[\sigma]}^{p, p}(\mathcal{D}, E)$. Since

$$
\begin{aligned}
\mathcal{P}_{\mathrm{v}} P \tilde{u} & =\left(I-\mathcal{P}_{\mathrm{dl}}\right) \tilde{u} \\
& =\sum_{\nu=0}^{\infty} \mathcal{P}_{\mathrm{dl}}^{\nu}\left(\mathcal{G}\left(\chi_{\mathcal{D}} f\right)+\mathcal{P}_{\mathrm{sl}} \tilde{u}_{1}\right)-\sum_{\nu=1}^{\infty} \mathcal{P}_{\mathrm{dl}}^{\nu}\left(\mathcal{G}\left(\chi_{\mathcal{D}} f\right)+\mathcal{P}_{\mathrm{sl}} \tilde{u}_{1}\right) \\
& =\mathcal{G}\left(\chi_{\mathcal{D}} f\right)+\mathcal{P}_{\mathrm{sl}} \tilde{u}_{1},
\end{aligned}
$$

we conclude that

$$
\begin{aligned}
\int_{\mathcal{D}}(P \tilde{u}, P v)_{x} d x & =h\left(\mathcal{P}_{\mathbf{v}} P \tilde{u}, v\right) \\
& =h\left(\mathcal{G}\left(\chi_{\mathcal{D}} f\right)+\mathcal{P}_{\mathrm{sl}} \tilde{u}_{1}, v\right)
\end{aligned}
$$

for any $v \in H_{[\sigma]}^{p, p}(\mathcal{D}, E)$, the first equality being due to Proposition 5.4.

Set $U=\mathcal{G}\left(\chi_{\mathcal{D}} f\right)+\mathcal{P}_{\mathrm{sl}} \tilde{u}_{1}$. Using the fact that $\mathcal{G}(x, y)$ is a two-sided fundamental solution for $\Delta$ in $\mathcal{D}^{\prime} \backslash \bar{\sigma}$, we get

$$
\begin{aligned}
\Delta U^{-} & =f, \\
\mathcal{E}\left(U^{-}\right) & =U^{+},
\end{aligned}
$$

where $U^{-}$and $U^{+}$stand for the restrictions of $U$ to $\mathcal{D}$ and $\mathcal{D}^{\prime} \backslash \overline{\mathcal{D}}$, respectively. Therefore, if $v$ is a $C^{\infty}$ section of $E$ vanishing in a neighbourhood of $\bar{\sigma}$, we 
have

$$
\begin{aligned}
\int_{\mathcal{D}}(P \tilde{u}, P v)_{x} d x & =\int_{\mathcal{D}}(f, v)_{x} d x+\int_{\partial \mathcal{D}}\left(n\left(P U^{-}\right)-n\left(P \mathcal{E}\left(U^{-}\right)\right), t(v)\right)_{x} d s \\
& =\int_{\mathcal{D}}(f, v)_{x} d x+\int_{\partial \mathcal{D}}\left(n\left(P U^{-}\right)-n\left(P U^{+}\right), t(v)\right)_{x} d s .
\end{aligned}
$$

By a jump theorem for the simple layer potential (cf. Theorem 10.1 .5 of [Tar95]), the difference $n\left(P U^{-}\right)-n\left(P U^{+}\right)$is equal to $\tilde{u}_{1}$ on $\partial \mathcal{D} \backslash \bar{\sigma}$. Therefore,

$$
\int_{\mathcal{D}}(P \tilde{u}, P v)_{x} d x=\int_{\mathcal{D}}(f, v)_{x} d x+\int_{\partial \mathcal{D}}\left(\tilde{u}_{1}, t(v)\right)_{x} d s,
$$

which gives (9.2) if combined with the fact that the functions $v \in C_{\text {loc }}^{\infty}(X, E)$ vanishing near $\bar{\sigma}$ are dense in $H_{[\sigma]}^{p, p}(\mathcal{D}, E)$. Thus, the problem (9.1) is solvable, which completes the proof.

Were the problem (9.1) first reduced to the particular case corresponding to $f=0$ and $u_{0}=0$, we would obtain yet another formula for approximate solutions of this problem.

Theorem 9.1 just amounts to saying that the operator $L$ bears the following two properties: 1) $L$ is injective, and 2) $\left(f, u_{0}, u_{1}\right) \in \operatorname{Ran} L$ is equivalent to the convergence of the series $(9.4)$ in $H^{p, p}(\mathcal{D}, E)$. Moreover, since the Zaremba problem is formally selfadjoint with respect to the Green formula for $\Delta$, it follows that the range of $L$ is dense in $H^{-p,-p}(\mathcal{D}, E) \oplus \mathfrak{D} \oplus \mathfrak{N}$.

Remark 9.2 If (9.1) is Fredholm, the series (9.4) converges in $H^{p, p}(\mathcal{D}, E)$, for each data $\left(f, u_{0}, u_{1}\right)$.

\section{Examples}

Example 10.1 Let $\sigma$ coincide with a connected component of $\partial \mathcal{D}$. Then, $\sigma$ is a $C^{\infty}$ closed hypersurface in $\mathcal{D}^{\prime}$. In this case the analysis above is carried out in the framework of usual (non-weighted) Sobolev spaces on $X$. For simplicity, we assume that $\sigma$ divides $\mathcal{D}^{\prime}$ into two domains $\mathcal{D}_{1}^{\prime}$ and $\mathcal{D}_{2}^{\prime}$. Write $\mathcal{G}_{1}(x, y)$ and $\mathcal{G}_{2}(x, y)$ for the Green functions of the Dirichlet problem for $\Delta$ in $\mathcal{D}_{1}^{\prime}$ and $\mathcal{D}_{2}^{\prime}$, respectively. Set

$$
\mathcal{G}(x, y)= \begin{cases}\mathcal{G}_{1}(x, y) & \text { if } \quad(x, y) \in \mathcal{D}_{1}^{\prime} \times \mathcal{D}_{1}^{\prime} \\ \mathcal{G}_{2}(x, y) & \text { if } \quad(x, y) \in \mathcal{D}_{2}^{\prime} \times \mathcal{D}_{2}^{\prime}\end{cases}
$$

then $\mathcal{G}(x, y)$, if extended by zero to the whole product $\left(\mathcal{D}^{\prime} \backslash \sigma\right) \times\left(\mathcal{D}^{\prime} \backslash \sigma\right)$, is easily seen to be the Green function of the Dirichlet problem for $\Delta$ in $\mathcal{D}^{\prime} \backslash \sigma$. On the other hand, the domain $\mathcal{D}$ belongs either to $\mathcal{D}_{1}^{\prime}$ or to $\mathcal{D}_{2}^{\prime}$. Since the 
potentials $\mathcal{P}_{\mathrm{dl}}, \mathcal{P}_{\mathrm{sl}}$ and $\mathcal{G}\left(\chi_{\mathcal{D}} \cdot\right)$ depend only on the restriction of $\mathcal{G}(x, y)$ to $\mathcal{D} \times \mathcal{D}$, it suffices to make use either of $\mathcal{G}_{1}(x, y)$ or of $\mathcal{G}_{2}(x, y)$ to define all the potentials. In particular, if $\sigma=\partial \mathcal{D}$, then we recover the results of Nacinovich and Shlapunov [NS96].

Example 10.2 Let $P$ be the gradient operator in $\mathbb{R}^{n}$. Then $-P^{*} P$ is the Laplace operator in $\mathbb{R}^{n}$. Denote by $G(x)$ the standard fundamental solution of convolution type for $-P^{*} P$, i.e.,

$$
-G(x)= \begin{cases}(1 / 2 \pi) \log |x| & \text { if } \quad n=2 \\ \left(1 /(2-n) \sigma_{n}\right)|x|^{2-n} & \text { if } \quad n>2\end{cases}
$$

$\sigma_{n}$ being the area of the unit sphere in $\mathbb{R}^{n}$. If $\mathcal{D}^{\prime}=\mathbb{R}^{n}, n \geq 2$, and $\sigma$ is the sphere $\partial B(0, R)$ in $\mathbb{R}^{n}$, then the reflection principle yields a Green function of the Dirichlet problem in either of the domains $B(0, R)$ and $\mathbb{R}^{n} \backslash \overline{B(0, R)}$ in the form

$$
\begin{aligned}
\mathcal{G}(x, y) & =G(x-y)-(R /|x|)^{n-2} G\left((R /|x|)^{2} x-y\right) \\
& =G(x-y)-(R /|y|)^{n-2} G\left(x-(R /|y|)^{2} y\right),
\end{aligned}
$$

the symmetry being easily verified. Taking $B_{0}=1$ we have $n(P u)=(\partial / \partial n) u$, the derivative of $u$ along the outward normal vector to the boundary. Thus, if $\mathcal{D}$ is a domain in $\mathbb{R}^{n}$, such that $\partial B(0, R)$ is a component of the boundary of $\mathcal{D}$, then

$$
\begin{aligned}
-\mathcal{P}_{\mathrm{dl}} u(x) & =\int_{\partial \mathcal{D}}(\partial / \partial n) \mathcal{G}(x, \cdot) u d s, \\
\mathcal{P}_{\mathrm{v}} f(x) & =\int_{\mathcal{D}} \sum_{j=1}^{n}\left(\partial / \partial y_{j}\right) \mathcal{G}(x, \cdot) f_{j} d y,
\end{aligned}
$$

for $x \in \mathbb{R}^{n} \backslash \partial \mathcal{D}$, where $u \in H^{1}(\mathcal{D})$ and $f \in L^{2}\left(\mathcal{D}, \mathbb{C}^{n}\right)$. 


\section{References}

[Ant66] Yu. T. Antokhin, An analytic approach to investigation of equations of the first type, Dokl. Akad. Nauk SSSR 167 (1966), no. 4, 727730 .

[C22] T. Carleman, Sur la resolution de certaines équations intégrales, Ark. Mat. Astr. Fys. 16 (1922), no. 26, 1-19.

[DW95] R. Duduchava and W. Wendland, The Wiener-Hopf method for systems of pseudodifferential equations with an application to crack problems, Integral Equ. and Operator Theory 23 (1995), 294-335.

[Esk73] G. I. Eskin, Boundary Value Problems for Elliptic Pseudodifferential Operators, Nauka, Moscow, 1973.

[G77] F. D. Gakhov, Boundary Value Problems, Nauka, Moscow, 1977.

[KGBB76] V. D. Kupradze, T. G. Gegeliya, M. O. Baskheleishvili, and T. V. Burchuladze, Three-Dimensional Problems of the Mathematical Theory of Elasticity and Thermoelasticity, Nauka, Moscow, 1976 .

[LRS80] M. M. Lavrent'ev, V. G. Romanov, and S. P. Shishatskii, Ill-posed Problems of Mathematical Physics and Analysis, Nauka, Moscow, 1980.

[NS96] M. Nacinovich and A. A. Shlapunov, On iterations of the Green integrals and their applications to elliptic differential complexes, Math. Nachr. 180 (1996), 243-286.

[NP91] S. A. Nazarov and B. A. Plamenevskii, Elliptic Boundary Value Problems in Domains with Piecewise Smooth Boundary, Nauka, Moscow, 1991.

[NCS90] D. Natroshvili, O. Chkadua, and E. Shargorodskii, Mixed problems for homogeneous anisotropic elastic media, Proceedings of I. Vekua Institute of Applied Math., Tbilisi State University 39 (1990), 133181.

[RST98] V. S. Rabinovich, B.-W. Schulze, and N. N. Tarkhanov, Boundary Value Problems in Cuspidal Wedges, Preprint 24, Univ. Potsdam, Potsdam, October 1998, 66 pp.

[RS82] S. Rempel and B.-W. Schulze, Index Theory of Elliptic Boundary Problems, Akademie-Verlag, Berlin, 1982. 
[Roi96] Ya. A. Roitberg, Elliptic Boundary Value Problems in Generalized Functions, Kluwer Academic Publishers, Dordrecht NL, 1996.

[Rom78] A. V. Romanov, Convergence of iterations of Bochner-Martinelli operator, and the Cauchy-Riemann system, Dokl. Akad. Nauk SSSR 242 (1978), no. 4, 780-783.

[Sch92] B.-W. Schulze, Crack problems in the edge pseudo-differential calculus, Applic. Analysis 45 (1992), 333-360.

[Sch98] B.-W. Schulze, Boundary Value Problems and Singular PseudoDifferential Operators, J. Wiley, Chichester, 1998.

[Sch99] B.-W. Schulze, Pseudo-Differential Calculus and Applications to Non-Smooth Configurations, Preprint 99/?, Univ. Potsdam, Potsdam, May 1999, 129 pp.

[Sh199] A. Shlapunov, Iterations of Self-Adjoint Operators and Their Applications to Elliptic Systems, Preprint 99/3, Univ. Potsdam, Potsdam, January 1999, $23 \mathrm{pp}$.

[Tar97] N. N. Tarkhanov, The Analysis of Solutions of Elliptic Equations, Kluwer Academic Publishers, Dordrecht, NL, 1997.

[Tar95] N. N. Tarkhanov, The Cauchy Problem for Solutions of Elliptic Equations, Akademie-Verlag, Berlin, 1995.

[Yos65] K. Yosida, Functional Analysis, Springer-Verlag, Berlin et al., 1965.

(B.-W. Schulze) Universität Potsdam, Institut für Mathematik, Postfach 6015 53, 14415 PotsDAM, Germany

E-mail address: schulze@math.uni-potsdam.de

(A. Shlapunov) Krasnoyarsk State University, pr. Svobodnyi, 79, 660041 Krasnoyarsk, Russia

E-mail address: shlapuno@math.kgu.krasnoyarsk.su

(N. Tarkhanov) Institute of Physics, Russian Academy of Sciences, Akademgorodok, 660036 Krasnoyarsk, Russia

E-mail address: tarkhan@math.uni-potsdam.de 